\title{
Attractor states and infrared scaling in de Sitter space
}

\author{
Paul R. Anderson ${ }^{1,2} *$, Wayne Eaker ${ }^{1 \dagger}$, Salman Habib ${ }^{2} \ddagger$, Carmen Molina-París ${ }^{2,3} \S$, and Emil Mottola ${ }^{2 * *}$ \\ 1 Department of Physics Wake Forest University, Winston-Salem, North Carolina, 27109 \\ 2 T-8, Theoretical Division, Los Alamos National Laboratory, Los Alamos, New Mexico, 87545 \\ ${ }^{3}$ Centro de Astrobiología, CSIC-INTA, Carretera de Ajalvir Km. 4, 28850 Torrejó n, Madrid, Spain
}

(LA-UR-00-52; May 23, 2000)

\begin{abstract}
The renormalized expectation value of the energy-momentum tensor for a scalar field with any mass $m$ and curvature coupling $\xi$ is studied for an arbitrary homogeneous and isotropic physical initial state in de Sitter spacetime. We prove quite generally that $\left\langle T_{a b}\right\rangle$ has a fixed point attractor behavior at late times, which depends only on $m$ and $\xi$, for any fourth order adiabatic state that is infrared finite. Specifically, when $m^{2}+\xi R>0,\left\langle T_{a b}\right\rangle$ approaches the Bunch-Davies de Sitter invariant value at late times, independently of the initial state. When $m=\xi=0$, it approaches instead the de Sitter invariant Allen-Folacci value. When $m=0$ and $\xi \geq 0$ we show that this state independent asymptotic value of the energy-momentum tensor is proportional to the conserved geometrical tensor ${ }^{(3)} H_{a b}$, which is related to the behavior of the quantum effective action of the scalar field under global Weyl rescaling. This relationship serves to generalize the definition of the trace anomaly in the infrared for massless, non-conformal fields. In the case $m^{2}+\xi R=0$, but $m$ and $\xi$ separately different from zero, $\left\langle T_{a b}\right\rangle$ grows linearly with cosmic time at late times. For most values of $m^{2}$ and $\xi$ in the tachyonic cases, $m^{2}+\xi R<0,\left\langle T_{a b}\right\rangle$ grows exponentially at late cosmic times for all physically admissable initial states.
\end{abstract}

\section{INTRODUCTION}

Quantum field theory in curved spacetimes does not contain in itself a unique specification of the quantum state of the system [1]. Even in Minkowski spacetime, where the existence of the Poincaré group singles out a special state, the Minkowski vacuum, it is certainly of interest to consider states that are non-invariant under Poincaré transformations, since they contain all the information about the physical excitations and dynamics of the theory. Such non-vacuum states are also necessary in a general initial value formulation of the back-reaction problem in both curved and flat spacetimes. In flat space the initial value problem for arbitrary physically allowable states has been formulated and studied for both QED and scalar $\Phi^{4}$ theory in the large $N$ limit, principally for time varying but spatially homogeneous mean fields [2.3].

The simplest situation in which the back-reaction problem can be studied in curved spacetime is that of a free scalar field in a spatially homogeneous and isotropic Robertson-Walker (RW) cosmology, where the geometry is characterized by just one non-trivial function of time. The wave equation for a free scalar field in such a geometry can be separated and expressed in terms of a complete set of time dependent mode functions. The general initial value problem is specified by giving initial data for this complete set at a given initial time. The back-reaction of the quantum scalar field(s) on the RW geometry can be studied then by constructing the renormalized expectation value of the energymomentum tensor $\left\langle T_{a b}\right\rangle$ of the field(s) and solving (numerically) the semi-classical Einstein equations, augmented by higher derivative terms required by renormalization [4 9]. As in the flat space examples, this semi-classical backreaction problem becomes exact in the large $N$ limit, with $N$ the number of identical scalar fields [10].

As a prelude to the dynamical back-reaction problem in cosmological spacetimes it is necessary to study non-vacuum states first in fixed RW backgrounds. The maximally symmetric de Sitter spacetime is of particular interest. Most previous work has focused on maximally symmetric $O(4,1)$ de Sitter invariant states or the special $O(4)$ invariant state found by Allen 11]. Since the universe is not globally $O(4,1)$ invariant, a more generic set of initial conditions, consistent only with RW symmetry and general principles of renormalization of $\left\langle T_{a b}\right\rangle$ is required for cosmology. The

\footnotetext{
*electronic address: anderson@wfu.edu

$\dagger$ electronic address: eaker@alumni.wfu.edu

‡electronic address: habib@lanl.gov

$\S$ electronic address: carmen@t6-serv.lanl.gov

** electronic address: emil@lanl.gov
} 
investigation of these much weaker requirements and specification of the general initial value problem for back-reaction calculations was initiated in Ref. [12].

In this paper we study the behavior of the renormalized $\left\langle T_{a b}\right\rangle$ for arbitrary physically admissable spatially homogeneous and isotropic states in a fixed de Sitter background. We argue in Section III that such states must be fourth order adiabatic states [1] that also possess an infrared finite two-point function. In de Sitter space the wave equation for free scalar fields can be solved exactly for arbitrary values of the mass and the curvature coupling. Its solutions depend only on the wave number $k$ of the mode and the parameter $\nu^{2}=\frac{9}{4}-m^{2} \alpha^{2}-12 \xi$, with $R=12 \alpha^{-2}$ the constant scalar curvature of de Sitter spacetime. For $\Re(\nu)<\frac{3}{2}$, corresponding to $m^{2}+\xi R>0$, we prove that for all UV and IR physically allowed initial states the renormalized value of $\left\langle T_{a b}\right\rangle$ at late times asymptotically approaches that of the Euclidean or Bunch-Davies de Sitter invariant state [13 16. The conformally invariant scalar field $\left(m=0, \xi=\frac{1}{6}\right)$ falls into this class.

The case $\nu=\frac{3}{2}$ corresponding to $m^{2} \alpha^{2}+12 \xi=0$ is more delicate. If $m$ and $\xi$ are separately zero (the massless, minimally coupled case), then we prove that the renormalized $\left\langle T_{a b}\right\rangle$ for all physically admissable states approaches the Allen-Folacci de Sitter invariant value [17 19]. Numerical evidence for this result was found previously in Ref. [12]. In this paper we provide an analytic proof that late time attractor behavior occurs for all physically admissable RW states, when $m^{2} \geq 0$ and $\xi \geq 0$. If $m^{2} \alpha^{2}+12 \xi=0$ but $m^{2}$ and $\xi$ are not separately zero (so that one of them is negative) we prove that $\left\langle T_{a b}\right\rangle$ grows linearly in RW comoving (cosmic) time without bound, and this asymptotic behavior is independent of the state of the field. Finally, and in contrast, in the case $\nu>\frac{3}{2}$, corresponding to $m^{2}+\xi R<0,\left\langle T_{a b}\right\rangle$ depends sensitively on the state and, for most values of $m$ and $\xi$, grows exponentially at late times for all states. This case is of considerably less physical relevance, since it corresponds to a tachyonic field theory with no stable vacuum state.

The asymptotic approach of $\left\langle T_{a b}\right\rangle$ to a de Sitter invariant form, independently of the lower symmetry of the initial data when $\Re(\nu) \leq \frac{3}{2}$ is a striking result. Certainly no such attractor behavior of $\left\langle T_{a b}\right\rangle$, independent of initial conditions occurs in Minkowski space for any mass. One may regard this result as a kind of cosmic "no hair" theorem for scalar quantum fields in de Sitter space. For $\Re(\nu)<\frac{3}{2}$ it is in accord with one's classical intuition that any initial energy density satisfying the weak energy condition $(\varepsilon+p>0)$ is redshifted away by the exponential de Sitter expansion, although as we will see, the redshifting of the quantum $\left\langle T_{a b}\right\rangle$ is not that of classical matter or radiation. At asymptotically late times what is left behind is a kind of frozen quantum vacuum energy "condensate," satisfying the de Sitter invariant equation of state $p=-\varepsilon$. This result justifies the choice of the Bunch-Davies vacuum in calculations of quantum fluctuations of free fields, i.e. without back-reaction, in a long-lived de Sitter expansion phase of inflationary cosmological models. For $\nu=\frac{3}{2}, m=\xi=0$, the approach of $\left\langle T_{a b}\right\rangle$ to the de Sitter invariant Allen-Folacci value is perhaps more surprising. As shown in Section IV one expects the leading order contribution of the modes to $\left\langle T_{a b}\right\rangle$ in this case to be constant in comoving time at late times. In fact this occurs if $m$ and $\xi$ are not separately zero for all the modes. However when $m$ and $\xi$ are both zero, the leading order contributions to $\left\langle T_{a b}\right\rangle$ of all the modes except the spatially homogeneous one, for an arbitrary physically admissable state have exactly zero coefficient, the subleading contributions redshift away, and we are left only with the with the de Sitter invariant AllenFolacci constant value at late times. The finite difference from the Bunch-Davies value may be attributed entirely to the constant behavior of the spatially homogeneous mode contributing to the vacuum energy condensate in de Sitter space.

In all those cases for which $\Re(\nu) \leq \frac{3}{2}$ when $\left\langle T_{a b}\right\rangle$ approaches a de Sitter invariant value at late times, the quantum expectation value loses all its initial state dependence and hence its asymptotic value must be determined purely by the background geometry. When the mass of the field vanishes, the existence of only one covariantly conserved local geometrical tensor of adiabatic order four in de Sitter space, namely ${ }^{(3)} H_{a b}$ given by Eq. (6.2) below, permits us to identify the asymptotic value of $\left\langle T_{a b}\right\rangle$ in the vacuum energy condensate with this tensor. Since ${ }^{(3)} H_{a b}$ cannot be derived by variation of a covariant local action, but corresponds instead to a certain well defined non-local term in the quantum effective action [20], the asymptotic vacuum energy condensate of the quantum field is determined by the global or extreme infrared properties of de Sitter space. The form of the non-local effective action is determined by the trace anomaly in conformally flat spacetimes. Since the approach of $\left\langle T_{a b}\right\rangle$ to a de Sitter invariant value occurs for all $\xi \geq 0$, the existence of this term in the effective action for massless fields is much more general than the strict definition of the trace anomaly in the conformally invariant case. Hence the asymptotic late time behavior of $\left\langle T_{a b}\right\rangle$ in de Sitter space can be used to define a generalized trace "anomaly" coefficient in the massless but non-conformally invariant cases, $\xi \neq \frac{1}{6}$. As we show by consideration of the covariant $\zeta$ function method 11, this coefficient is exactly the same as that which determines the infrared response of the vacuum condensate to global Weyl rescalings. Hence the significance of the state-independence of the vacuum energy condensate in de Sitter space is that it determines certain conformal properties of non-conformal field theories in the extreme infrared.

The paper is organized as follows. In Section [1] we give the expectation value of the energy-momentum tensor as a mode sum for an arbitrary homogeneous and isotropic, physically admissable state with a non-zero initial particle 
number of the scalar field. In Section III we analyze the late time behavior of expectation value of the energymomentum tensor in de Sitter space in flat spatial sections and show that it approaches the de Sitter invariant Bunch-Davies value for all $\Re(\nu)<\frac{3}{2}$, independently of the initial state. In Section $\left[\mathrm{V}\right.$ we analyze the limit $\Re(\nu) \rightarrow \frac{3}{2}$ in closed spatial sections in order to keep careful track of the spatially homogeneous mode in a discrete basis, and show how the Allen-Folacci fixed point at late times is obtained for the massless minimally coupled field. We also investigate the asymptotic behavior of the energy-momentum tensor for arbitrary mass and curvature coupling when $\nu \geq \frac{3}{2}$. In Section $\mathrm{V}$ we illustrate the analytic results with numerical studies, investigating in particular the interesting case when $\nu$ is slightly smaller than $\frac{3}{2}$. We find that for many states when $\nu$ is only slightly smaller than $\frac{3}{2}$ the energymomentum tensor first approaches the Allen-Folacci value and only much later approaches the Bunch-Davies value. In Section VI we consider the geometric significance of the state independent asymptotic behavior of $\left\langle T_{a b}\right\rangle$, relating it to the quantum effective action which determines the behavior of $S_{\text {eff }}$ under global Weyl scaling and providing the generalization of the trace "anomaly" in the infrared, for $\xi \neq \frac{1}{6}$. Section VII contains some discussion and final conclusions. There are two Appendices. Appendix A completes the proof of the Bunch-Davies attractor behavior in the cases of integer and pure imaginary $\nu$, while Appendix B contains a discussion of the simple harmonic oscillator in the limit of vanishing frequency, which shares many features with the spatially constant mode in de Sitter spacetime.

\section{SCALAR FIELD IN A RW BACKGROUND}

The metric for a general RW spacetime can be written in conformal time $\eta$ in the form

$$
\mathrm{d} s^{2}=a^{2}(\eta)\left(-\mathrm{d} \eta^{2}+\frac{\mathrm{d} r^{2}}{1-\kappa r^{2}}+r^{2} \mathrm{~d} \Omega^{2}\right)
$$

Here $a(\eta)$ is the scale factor and $\kappa=0,+1,-1$ corresponds to the cases of flat, spherical, and hyperbolic spatial sections, respectively. Throughout we use units such that $\hbar=c=1$ and the Misner, Thorne, and Wheeler 21 conventions for the curvature tensors, $R_{b c d}^{a}=\Gamma_{b d, c}^{a}-\ldots$ and $R_{a b}=R_{a c b}^{c}$.

We consider in this paper a free quantum scalar field $\Phi$ with the quadratic action

$$
S=-\frac{1}{2} \int \mathrm{d}^{4} x \sqrt{-g}\left[\left(\nabla_{a} \Phi\right) g^{a b}\left(\nabla_{b} \Phi\right)+m^{2} \Phi^{2}+\xi R \Phi^{2}\right]
$$

where $\nabla_{a}$ denotes the covariant derivative, $R$ is the scalar curvature, and $g \equiv \operatorname{det}\left(g_{a b}\right)$. The mass $m$ and curvature coupling $\xi$ are allowed to have any real value. The wave equation for $\Phi$ obtained by varying this action is

$$
\left[-.09 .09+m^{2}+\xi R\right] \Phi(\eta, \mathbf{x})=\left[\frac{1}{a^{4}} \frac{\partial}{\partial \eta}\left(a^{2} \frac{\partial}{\partial \eta}\right)-\frac{1}{a^{2}} \Delta^{(3)}+m^{2}+\xi R\right] \Phi=0
$$

with $\Delta^{(3)}$ the covariant spatial Laplacian. For spacetimes with the metric 2.1 the field $\Phi$ can be expanded as a mode sum in the form, [1]

$$
\Phi(\eta, \mathbf{x})=\frac{1}{a(\eta)} \int \mathrm{d} \tilde{\mu}(\mathbf{k})\left[a_{\mathbf{k}} Y_{\mathbf{k}}(\mathbf{x}) \psi_{k}(\eta)+a_{\mathbf{k}}^{\dagger} Y_{\mathbf{k}}^{*}(\mathbf{x}) \psi_{k}^{*}(\eta)\right]
$$

where the integration measure is given by

$$
\int \mathrm{d} \tilde{\mu}(\mathbf{k}) \equiv \begin{cases}\int \mathrm{d}^{3} \mathbf{k} & \text { if } \kappa=0 \\ \int_{0}^{\infty} \mathrm{d} k \sum_{l, m} & \text { if } \kappa=-1 \\ \sum_{k, l, m} & \text { if } \kappa=+1\end{cases}
$$

and the spatial part of the mode functions $Y_{\mathbf{k}}(\mathbf{x})$ obeys the equation

$$
-\Delta^{(3)} Y_{\mathbf{k}}(\mathbf{x})=\left(k^{2}-\kappa\right) Y_{\mathbf{k}}(\mathbf{x})
$$

with $k=1,2, \ldots$ in the case of closed spatial sections, $\kappa=+1$. The time dependent part of the mode functions $\psi_{k}$ obeys the equation

$$
\psi_{k}^{\prime \prime}+\left[k^{2}+m^{2} a^{2}+\left(\xi-\frac{1}{6}\right) a^{2} R\right] \psi_{k}=0
$$


where primes denote derivatives with respect to the conformal time variable $\eta$, and the scalar curvature in a general RW spacetime is given by

$$
R=6\left(\frac{a^{\prime \prime}}{a^{3}}+\frac{\kappa}{a^{2}}\right)
$$

For the quantum field to satisfy the canonical commutation relations, the creation and annihilation operators are required to obey the commutation relations $\left[a_{\mathbf{k}}, a_{\mathbf{k}^{\prime}}^{\dagger}\right]=\delta_{\mathbf{k k}^{\prime}}$, whereupon the $\psi_{k}$ must obey the Wronskian condition

$$
\psi_{k} \psi_{k}^{* \prime}-\psi_{k}^{*} \psi_{k}^{\prime}=i
$$

The components of the unrenormalized energy-momentum tensor (energy density and trace) are given by [22]

$$
\begin{aligned}
& \varepsilon_{u}=-\left\langle T^{0}{ }_{0}\right\rangle_{u}=\frac{1}{4 \pi^{2} a^{4}} \int \mathrm{d} \mu(k)\left(2 n_{k}+1\right)\left\{\left|\psi_{k}^{\prime}\right|^{2}+\left(k^{2}+m^{2} a^{2}\right)\left|\psi_{k}\right|^{2}\right. \\
&\left.+(6 \xi-1)\left[\frac{a^{\prime}}{a}\left(\psi_{k} \psi_{k}^{* \prime}+\psi_{k}^{*} \psi_{k}^{\prime}\right)-\left(\frac{a^{\prime 2}}{a^{2}}-\kappa\right)\left|\psi_{k}\right|^{2}\right]\right\}
\end{aligned}
$$

and

$$
\begin{aligned}
-\varepsilon_{u}+3 p_{u}= & \langle T\rangle_{u}=\frac{1}{2 \pi^{2} a^{4}} \int \mathrm{d} \mu(k)\left(2 n_{k}+1\right)\left\{-m^{2} a^{2}\left|\psi_{k}\right|^{2}+(6 \xi-1)\left[-\left|\psi_{k}^{\prime}\right|^{2}+\frac{a^{\prime}}{a}\left(\psi_{k} \psi_{k}^{* \prime}+\psi_{k}^{*} \psi_{k}^{\prime}\right)\right]\right. \\
& \left.\left.+(6 \xi-1)\left[k^{2}+m^{2} a^{2}+\left(\frac{a^{\prime \prime}}{a}-\frac{a^{\prime 2}}{a^{2}}\right)+\left(\xi-\frac{1}{6}\right) a^{2} R\right]\left|\psi_{k}\right|^{2}\right]\right\} .
\end{aligned}
$$

where we have allowed an arbitrary number of particles in the initial state, $n_{k}=\left\langle a_{\mathbf{k}}^{\dagger} a_{\mathbf{k}}\right\rangle$, and the scalar measure $\mathrm{d} \mu(k)$ is given by

$$
\int \mathrm{d} \mu(k) \equiv \begin{cases}\int_{0}^{\infty} \mathrm{d} k k^{2} & \text { if } \kappa=0,-1, \\ \sum_{1}^{\infty} k^{2} & \text { if } \kappa=+1 .\end{cases}
$$

As we are considering spatially homogeneous and isotropic initial states (consistent with the RW symmetry), $n_{k}$ depends only on the magnitude $k$ of the spatial wave vector $\mathbf{k}$. Expectation values of the bilinears $\left\langle a_{\mathbf{k}} a_{\mathbf{k}}\right\rangle$ and $\left\langle a_{\mathbf{k}}^{\dagger} a_{\mathbf{k}}^{\dagger}\right\rangle$ in a general state need not be considered since they can be removed by a time-independent Bogoliubov transformation at the initial time [2]. Hence these initial state correlations may be parameterized instead by the initial data on the mode functions $\psi_{k}$, together with the non-negative set of $n_{k}$, with no loss of generality.

Since the expectation value of the unrenormalized energy-momentum tensor $\left\langle T_{a b}\right\rangle_{u}$ is quartically divergent, a procedure for defining a finite, renormalized expectation value must be given. We will follow the adiabatic regularization method 23 26. In this method the renormalization counterterms are constructed using a fourth order WKB expansion for the mode functions. We denote these counterterms by $\left\langle T_{a b}\right\rangle_{a d}$. They are given in Refs. 222] and [27]. The renormalized energy-momentum tensor is then

$$
\left\langle T_{a b}\right\rangle_{r e n}=\left\langle T_{a b}\right\rangle_{u}-\left\langle T_{a b}\right\rangle_{a d} .
$$

This subtraction scheme is not manifestly covariant in form, since space and time are treated quite differently. However, adiabatic regularization is equivalent to a covariant point splitting procedure in which the points are split only in the spacelike hypersurface of constant $\eta$ [27,28], and the value of the renormalized $\left\langle T_{a b}\right\rangle$ obtained by this procedure is the same as in a strictly covariant one. Hence this subtraction procedure does correspond to adjustment of counterterms to the quantum effective action, and $\left\langle T_{a b}\right\rangle_{r e n}$ is covariantly conserved. As discussed in detail in Ref. [27] the adiabatic terms in all cases consist of an integral rather than a sum over $k$. The reason is that subtraction corresponds to purely local counterterms in the effective action, and thus must be independent of the global compactness or non-compactness of the spatial sections.

For an arbitrary homogeneous and isotropic state to be physically admissible the renormalized energy-momentum tensor defined by the adiabatic order four subtractions in (2.10) must be both ultraviolet and infrared finite. In a general RW spacetime, ultraviolet finiteness for a field with non-conformal coupling to the scalar curvature requires that the particular solution of the mode equation (2.6) in a general physical state must match the fourth order adiabatic form at large $k$, with the deviations from the fourth order WKB form falling faster than $k^{-4}$. Likewise the initial number $n_{k}$ must fall faster than $k^{-4}$ at large $k$, for the mode sums (integrals) to be ultraviolet convergent. This 
is equivalent to the requirement that the two-point function of the scalar field have the vacuum Hadamard form 29 32] to sufficiently high order in the short distance expansion as the points approach one another. As long as there are two linearly independent complex oscillatory solutions to the equation (2.6), the Wronskian normalization condition (2.8) can be imposed and the state will be free of any infrared divergences. However, when $m^{2}+\xi R \rightarrow 0$ for some low $k$ in de Sitter space no such complex oscillatory solutions to (2.6) exist and the infrared finiteness requirement on the both the energy-momentum tensor and the two-point function of the physical state becomes non-trivial, as we discuss in detail in Section IV.

A useful variation of the method of adiabatic regularization has been developed by two of us [33]. In this method one first computes a quantity $\left\langle T_{a b}\right\rangle_{d}$, obtained by expanding the adiabatic counterterms $\left\langle T_{a b}\right\rangle_{a d}$ in inverse powers of $k$ and truncating at order $k^{-3}$. The same renormalized energy-momentum tensor defined in Eq. (2.10) is separated into the sum of two finite terms by adding and subtracting the simplified form of the divergent counterterms $\left\langle T_{a b}\right\rangle_{d}$

$$
\begin{aligned}
\left\langle T_{a b}\right\rangle_{r e n} & =\left\langle T_{a b}\right\rangle_{n}+\left\langle T_{a b}\right\rangle_{a n}, \\
\left\langle T_{a b}\right\rangle_{n} & =\left\langle T_{a b}\right\rangle_{u}-\left\langle T_{a b}\right\rangle_{d}, \\
\left\langle T_{a b}\right\rangle_{a n} & =\left\langle T_{a b}\right\rangle_{d}-\left\langle T_{a b}\right\rangle_{a d} .
\end{aligned}
$$

The full expressions for $\left\langle T_{a b}\right\rangle_{d}$ and $\left\langle T_{a b}\right\rangle_{a n}$ are given in Ref. 33] for a general RW spacetime. The advantage of this splitting is that $\left\langle T_{a b}\right\rangle_{n}$ and $\left\langle T_{a b}\right\rangle_{a n}$ are separately conserved, and moreover, $\left\langle T_{a b}\right\rangle_{a n}$ may be computed analytically in terms of the scale factor $a(\eta)$ and its derivatives [33]. Thus the state dependence of the renormalized $\left\langle T_{a b}\right\rangle_{r e n}$ resides completely in $\left\langle T_{a b}\right\rangle_{n}$, which can be computed numerically.

\section{THE BUNCH-DAVIES ATTRACTOR FOR $\Re(\nu)<\frac{3}{2}$}

We restrict our consideration henceforth to the particular maximally symmetric RW background of de Sitter space. The geometry of de Sitter spacetime can be described in a number of different coordinate systems. If $\kappa=0$ the spatial sections are flat and the scale factor is

$$
a(\eta)=-\frac{\alpha}{\eta}, \quad-\infty<\eta<0, \quad \kappa=0,
$$

with $\alpha$ a real, positive constant, and $R=12 \alpha^{-2}$. If $\kappa=+1$ then the scale factor is

$$
a(\eta)=\alpha \sec \eta, \quad-\frac{\pi}{2}<\eta<\frac{\pi}{2}, \quad \kappa=+1,
$$

which is equivalent to $a(\eta)=\alpha \csc \eta$ with $0<\eta<\pi$. To simplify the notation we will generally use dimensionless units where $\alpha=1$ and $R=12$, restoring the dimensionful quantities when it is instructive to do so.

The asymptotic behavior of the energy-momentum tensor does not depend on $\kappa$ so the bulk of the analysis will be carried out in the flat $(\kappa=0)$ coordinates. However, when we turn to the massless, minimally coupled limit in the next section, it will become useful to have a discrete $k$ basis in order to separate out the $k=1$ spatially homogeneous mode explicitly, since it is the most infrared sensitive. No confusion should be caused by our use of the same symbol $\eta$ for conformal time in both cases of flat and closed spatial sections, since we make use of only the $\kappa=0$ coordinates in this Section and only the $\kappa=+1$ coordinates in the next Section. We will not make use of the spatially open $(\kappa=-1)$ coordinates in this paper.

For the case of Eq. (3.1) the general solution to the mode equation can be written as 16] 1

$$
\psi_{k}(\eta)=\frac{1}{2}(-\pi \eta)^{\frac{1}{2}} e^{\frac{i \nu \pi}{2}}\left[c_{1}(k) H_{\nu}^{(1)}(-k \eta)+c_{2}(k) H_{\nu}^{(2)}(-k \eta)\right],
$$

where the $H_{\nu}^{(1),(2)}$ are Hankel functions and

$$
\nu^{2} \equiv \frac{9}{4}-m^{2} \alpha^{2}-12 \xi \equiv-\gamma^{2} .
$$

\footnotetext{
${ }^{1}$ In Ref. 16 the arguments of the Hankel functions are given as $k \eta$ rather than $-k \eta$. We have chosen to use non-negative arguments to avoid complications that result from the fact that these functions have branch cuts along the negative real axis.
} 
The latter notation is useful in the case $\nu^{2}<0$ so that $\nu=i \gamma$ is purely imaginary. When $\nu^{2}>0$ we will choose $\nu$ to be the positive root of (3.4). From Eq. (3.3) we see that solutions to the mode equation in de Sitter space depend on $m$ and $\xi$ only through their dependence on the parameter $\nu$. Note that because of the minus sign in the arguments of the Hankel functions, it is the function $H_{\nu}^{(1)}$ that corresponds to a positive frequency mode in the large $k$ limit. The normalization of the mode function in (3.3) has been chosen so that the Wronskian condition (2.8) becomes simply

$$
\left|c_{1}(k)\right|^{2}-\left|c_{2}(k)\right|^{2}=1 .
$$

The Bunch-Davies (BD) state is defined by the choice, $c_{1}=1$ and $c_{2}=0$ (with $n_{k}=0$ ) for all $k$. The renormalized energy-momentum tensor in the BD state is given by [15,16]

$$
\begin{aligned}
\left\langle T_{a b}\right\rangle_{B D} & =-\frac{g_{a b}}{64 \pi^{2}}\left\{m^{2}\left[m^{2}+\left(\xi-\frac{1}{6}\right) R\right]\left[\psi\left(\frac{3}{2}+\nu\right)+\psi\left(\frac{3}{2}-\nu\right)-\log \left(\frac{12 m^{2}}{R}\right)\right]\right. \\
& \left.-m^{2}\left(\xi-\frac{1}{6}\right) R-\frac{1}{18} m^{2} R-\frac{1}{2}\left(\xi-\frac{1}{6}\right)^{2} R^{2}+\frac{R^{2}}{2160}\right\}
\end{aligned}
$$

where $\psi(z)=\frac{d \log \Gamma(z)}{d z}$ is the digamma function.

That this finite value of $\left\langle T_{a b}\right\rangle_{B D}$ coincides with the renormalized $\left\langle T_{a b}\right\rangle_{r e n}$ defined by the adiabatic subtraction in (2.10) follows from the fact that the BD state is an allowed fourth order adiabatic state. This may be checked by comparing the asymptotic expansion of the exact $\mathrm{BD}$ mode function, $\frac{(-\pi \eta)^{\frac{1}{2}}}{2} H_{\nu}^{(1)}(-k \eta)$, for large $k$ with the fourth order adiabatic mode function

$$
\psi_{k}^{(4)}(\eta)=\frac{1}{\sqrt{2 W_{k}^{(4)}}} \exp \left(-i \int^{\eta} W_{k}^{(4)}\left(\eta^{\prime}\right) \mathrm{d} \eta^{\prime}\right),
$$

with $W_{k}^{(4)}(\eta)$ the fourth order adiabatic frequency. It is given explicitly in de Sitter space in flat conformal time coordinates by

$$
W_{k}^{(4)}(\eta)=k+\frac{1}{2 k \eta^{2}}\left(\frac{1}{4}-\nu^{2}\right)-\frac{1}{8 k^{3} \eta^{4}}\left(\frac{1}{4}-\nu^{2}\right)\left(\frac{25}{4}-\nu^{2}\right)+\mathcal{O}\left(\frac{1}{k^{5}}\right)
$$

up to the required order at large $k$.

For the general state with $c_{2} \neq 0$ to remain fourth order adiabatic, we must have for large values of $k$

$$
c_{2}(k)=\frac{C(k)}{k^{4}},
$$

for some complex function $C(k)$ which vanishes in the limit $k \rightarrow \infty$. This condition is necessary for an arbitrary (spatially homogeneous) state to posses a finite energy-momentum tensor after the fourth order adiabatic subtraction defined by $(2.10)$. Likewise the same condition of finite $\left\langle T_{a b}\right\rangle$ requires us to restrict the average number of particles $\left\langle a_{\mathbf{k}}^{\dagger} a_{\mathbf{k}}\right\rangle=n_{k}$ by

$$
n_{k}=\frac{N(k)}{k^{4}}
$$

for some real function $N(k)$ which vanishes in the limit $k \rightarrow \infty$. The two ultraviolet conditions

$$
\lim _{k \rightarrow \infty}|C(k)|=\lim _{k \rightarrow \infty} N(k)=0,
$$

on the physically allowed states guarantee that the Green's function for the scalar field is locally of the Hadamard form [29 32, and that the divergences of $\left\langle T_{a b}\right\rangle$ match those of the fourth order adiabatic vacuum, and are removed by the adiabatic subtraction procedure. We will call any state which satisfies the conditions (3.9), (3.10), and (3.11), together with the Wronskian condition (3.5), a UV admissible physical state 2 .

\footnotetext{
${ }^{2}$ In fact, the requirement of fourth order adiabatic states is slightly more restrictive than UV finiteness of the energy-momentum tensor in de Sitter space, since $C(k)$ can go to a non-zero constant at large $k$ and the mode sums still converge. This is associated with the vanishing of the tensor, ${ }^{(1)} H_{a b}$, defined in Eq. (6.1) in de Sitter space. If the field is non-conformally coupled, a state with $C(k) \rightarrow$ constant would lead to a divergent energy-momentum tensor if the spacetime is not exactly de Sitter. If the field is conformally coupled and the spacetime is not exactly homogeneous and isotropic, then the energy-momentum tensor would again be divergent. Thus the most general physically acceptable UV states are fourth order adiabatic states.
} 
We shall require also that the arbitrary physical state possess a two-point function and energy-momentum tensor which are free of any infrared divergences. Because the canonical dimension of $\left\langle T_{a b}\right\rangle$ is four whereas that of $\left\langle\Phi(x) \Phi\left(x^{\prime}\right)\right\rangle$ is two, the conditions (3.9) and (3.10) which require finiteness of $\left\langle T_{a b}\right\rangle_{r e n}$ are more restrictive in the UV, whereas the condition of finiteness of $\left\langle\Phi(x) \Phi\left(x^{\prime}\right)\right\rangle$ is more restrictive in the IR. These two sets of conditions will be sufficient to demonstrate that the energy-momentum tensor for any UV and IR admissible physical state approaches the BD value at late times for $\Re(\nu)<\frac{3}{2}$.

To understand why such a result is to be expected and outline the more detailed proof which we give below, let us observe that at late times $\eta \rightarrow 0^{-}$, the general state mode function (3.3) behaves like

$$
\psi_{k} \sim(-\eta)^{\frac{1}{2}-\nu} \sim a^{\nu-\frac{1}{2}} .
$$

Substituting this into $(2.9 \mathrm{a})$ and $(2.9 \mathrm{~b})$ shows that to leading order at late times the contributions to the mode sums of the unrenormalized energy-momentum tensor behave like $(-\eta)^{3-2 \nu} \sim a^{2 \nu-3}$ for $\nu$ real. Since the renormalization counterterms are state independent [22], the state dependent terms are the same in the unrenormalized and renormalized energy-momentum tensor. One can perform all the UV renormalization in the BD state at a fixed time and collect the remaining finite state dependent terms which are unaffected by the subtraction procedure, and they all fall off at least as fast as $(-\eta)^{3-2 \nu}$ as $\eta \rightarrow 0^{-}$for $\Re(\nu)<\frac{3}{2}$.

These remaining finite state dependent terms in the energy density and trace are expressible as integrals over the wave number $k$ with the general form

$$
I(\eta)=\int_{0}^{\infty} \frac{\mathrm{d} k}{k} R(k) S(-k \eta),
$$

where $R(k)$ is one of the four state dependent, but time independent functions

$$
\begin{array}{r}
\left|c_{2}(k)\right|^{2}\left(1+2 n_{k}\right), \\
\Re\left[c_{1}(k) c_{2}(k)\right]\left(1+2 n_{k}\right), \\
\Im\left[c_{1}(k) c_{2}(k)\right]\left(1+2 n_{k}\right), \\
n_{k},
\end{array}
$$

and $S(z=-k \eta)$ is a product of the state independent Bessel functions and their derivatives. An explicit basis for the twelve products of Bessel functions $S_{i}(z)$ for $i=1, \ldots, 12$ which appear in the integrals is given in Table 1 . The essential point is that all the state dependent mode integrals of the form (3.13) are uniformly convergent for all $\eta$ (including $\eta=0$ ) at both their lower limit, $k=0$, and their upper limit $k=\infty$, due to the IR and UV finiteness of the state. Hence the limit of $\eta \rightarrow 0^{-}$can be taken inside the integral over $k$. Since, as Table 1 shows, all the $S_{i}(z)$ behave like

$$
S_{i}(z) \rightarrow s_{i, 0} z^{\beta_{i}}, \quad \text { with } \quad \beta_{i}>0,
$$

as $z \rightarrow 0$, for $\Re(\nu)<\frac{3}{2}$, it follows that

$$
\lim _{\eta \rightarrow 0^{-}} I(\eta)=\int_{0}^{\infty} \frac{\mathrm{d} k}{k} R(k) \lim _{\eta \rightarrow 0^{-}} S(-k \eta)=0,
$$

and all the state dependent contributions to $\left\langle T_{a b}\right\rangle_{r e n}$ vanish at late times.

The validity of bringing the limit inside the integral depends on the uniform convergence of the integral at both its upper and lower limits. In the form (3.13) the behavior of the $S(-k \eta)$ factor at small arguments (and the absence of any IR divergence from the $R(k)$ factor) clearly guarantees the uniform convergence at the lower limit. However, the change of variables $z=-k \eta$ brings the integral (3.13) into the form

$$
I(\eta)=\int_{0}^{\infty} \frac{\mathrm{d} z}{z} R\left(\frac{z}{-\eta}\right) S(z) .
$$

In this form it is clear that the uniform convergence of the integral at its upper limit is guaranteed by the falloff of the state dependent mode functions at large $k$, namely

$$
\lim _{k \rightarrow \infty} R(k)=\lim _{\eta \rightarrow 0^{-}} R\left(\frac{z}{-\eta}\right)=0 .
$$


Equations (3.15) and (3.18) guarantee that both the IR and UV contributions go to zero as $\eta \rightarrow 0^{-}$for $\Re(\nu)<\frac{3}{2}$. In fact, we can go one step further by using the information from the fourth order adiabatic nature of the state

$$
\lim _{k \rightarrow \infty} k^{4} R(k)=0,
$$

to conclude that the UV contribution to the integral from $z \sim 1$, and very large $k \sim(-\eta)^{-1}$ in (3.17) falls faster than $(-\eta)^{4}$ as $\eta \rightarrow 0^{-}$. This is faster than the IR contribution which falls only as $(-\eta)^{\beta_{i}}$ if $\beta_{i} \leq 4$. If $\beta_{i}>4$ then both the IR and UV contributions fall faster than $(-\eta)^{4}$. Hence, the $S_{i}(z)$ for which $\beta_{i}>4$ are subdominant at late times. Thus we conclude that the leading order state dependent terms at late times are those with the smallest $\beta_{i}$. These give an IR dominant, (i.e. finite $k, z \ll 1$ ) contribution of the form,

$$
I(\eta) \rightarrow(-\eta)^{\beta_{i}} \int_{0}^{\infty} \mathrm{d} k k^{\beta_{i}-1} R(k) \rightarrow(-\eta)^{3-2 \nu} \int_{0}^{\infty} \mathrm{d} k k^{2-2 \nu} R(k),
$$

as $\eta \rightarrow 0^{-}$. This last integral is guaranteed to converge at its upper limit for all $\Re(\nu) \geq-\frac{1}{2}$, and in particular for $0 \leq \Re(\nu) \leq \frac{3}{2}$ by (3.19). Hence for all $\Re(\nu)<\frac{3}{2}$ the state dependent contributions go to zero as $a^{2 \nu-3}$ at late times. The limiting case when $\nu \rightarrow \frac{3}{2}$ is special because then the state dependent IR contributions apparently does not go to zero at late times. This case will be considered separately in the next Section.

We will now make the proof more explicit by giving the form of all of the state dependent terms of the scalar field in de Sitter space, and analyzing the IR and UV contributions in detail. If we make use of Eq. (3.5), we find that in an arbitrary state

$$
\left\langle T_{a b}\right\rangle_{r e n}=\left\langle T_{a b}\right\rangle_{B D}+\left\langle T_{a b}\right\rangle_{S D}
$$

where $\left\langle T_{a b}\right\rangle_{S D}$ is the finite state dependent term, depending on the coefficients $c_{1}(k), c_{2}(k)$, and $n_{k}$, which may be expressed as an integral over the wave number $k$ in the form

$$
\left\langle T_{a b}\right\rangle_{S D}=\frac{1}{4 \pi^{2}} \int_{0}^{\infty} \mathrm{d} k I_{a b}(k, \eta) .
$$

The explicit expressions for the integrand $I_{a b}$ depend on whether $\nu$ is real and not an integer, real and an integer, or pure imaginary, although the result will be the same for all $\Re(\nu)<\frac{3}{2}$. In the rest of this Section we restrict our discussion to the case where $\nu$ is real and not equal to an integer. The cases of integer and imaginary values of $\nu$ are covered in Appendix A. For real $\nu$ after some regrouping of terms we have

$$
\begin{aligned}
I_{0}^{0}= & A_{1}(k)\left[S_{1}+\frac{1}{4}\left(9+4 m^{2}-48 \xi\right) S_{4}-\frac{3}{2}(4 \xi-1) S_{7}+S_{10}\right] \\
& +A_{2}(k)\left[S_{2}+\frac{1}{4}\left(9+4 m^{2}-48 \xi\right) S_{5}-\frac{3}{2}(4 \xi-1) S_{8}+S_{11}\right] \\
& +A_{3}(k)\left[S_{3}+\frac{1}{4}\left(9+4 m^{2}-48 \xi\right) S_{6}-\frac{3}{2}(4 \xi-1) S_{9}+S_{12}\right], \quad \text { and } \\
I= & A_{1}(k)\left[-2(6 \xi-1) S_{1}-\frac{1}{2}\left(9+8(3 \xi-1) m^{2}-102 \xi+288 \xi^{2}\right) S_{4}+3(6 \xi-1) S_{7}+2(6 \xi-1) S_{10}\right] \\
& +A_{2}(k)\left[-2(6 \xi-1) S_{2}-\frac{1}{2}\left(9+8(3 \xi-1) m^{2}-102 \xi+288 \xi^{2}\right) S_{5}+3(6 \xi-1) S_{8}+2(6 \xi-1) S_{11}\right] \\
& +A_{3}(k)\left[-2(6 \xi-1) S_{3}-\frac{1}{2}\left(9+8(3 \xi-1) m^{2}-102 \xi+288 \xi^{2}\right) S_{6}+3(6 \xi-1) S_{9}+2(6 \xi-1) S_{12}\right],
\end{aligned}
$$

with

$$
\begin{aligned}
A_{1}(k)= & -\frac{\pi}{2 k}\left[\csc ^{2}(\nu \pi)\left(\left(1+2 n_{k}\right)\left|c_{2}\right|^{2}+n_{k}\right)+\frac{1}{2}\left(1-\cot ^{2}(\nu \pi)\right)\left(1+2 n_{k}\right)\left(c_{1} c_{2}^{*}+c_{1}^{*} c_{2}\right)\right. \\
& \left.+\frac{i}{2} \cot (\nu \pi)\left(1+2 n_{k}\right)\left(c_{1} c_{2}^{*}-c_{1}^{*} c_{2}\right)\right], \\
A_{2}(k)= & \frac{\pi}{k}\left[\cot (\nu \pi) \csc (\nu \pi)\left(\left(1+2 n_{k}\right)\left|c_{2}\right|^{2}+n_{k}\right)-\frac{1}{2} \cot (\nu \pi) \csc (\nu \pi)\left(1+2 n_{k}\right)\left(c_{1} c_{2}^{*}+c_{1}^{*} c_{2}\right)\right.
\end{aligned}
$$




$$
\begin{aligned}
& \left.+\frac{i}{2} \csc (\nu \pi)\left(1+2 n_{k}\right)\left(c_{1} c_{2}^{*}-c_{1}^{*} c_{2}\right)\right], \\
A_{3}(k)=- & \frac{\pi}{2 k}\left[\csc ^{2}(\nu \pi)\left(\left(1+2 n_{k}\right)\left|c_{2}\right|^{2}+n_{k}\right)-\frac{1}{2} \csc ^{2}(\nu \pi)\left(1+2 n_{k}\right)\left(c_{1} c_{2}^{*}+c_{1}^{*} c_{2}\right)\right],
\end{aligned}
$$

and the $S_{i}(-k \eta)$ composed of various products of $J_{\nu}(-k \eta), J_{-\nu}(-k \eta)$ and their derivatives. Making use of the general formula for the product of two Bessel functions [34],

$$
J_{\mu}(z) J_{\nu}(z)=\sum_{p=0}^{\infty} \frac{(-1)^{p}\left(\frac{z}{2}\right)^{\nu+\mu+2 p} \Gamma(\nu+\mu+2 p+1)}{p ! \Gamma(\nu+\mu+p+1) \Gamma(\nu+p+1) \Gamma(\mu+p+1)},
$$

we can expand the $S_{i}$ in power series of the form,

$$
S_{i}(z)=\sum_{p=0}^{\infty} s_{i, p} z^{2 p+\beta_{i}},
$$

with $z=-k \eta$. The explicit expressions for $\beta_{i}$ and $S_{i}$ for the case of real, non-integer $\nu$ are given in Table 1 .

\begin{tabular}{|r||c|c|}
\hline$i$ & $\beta_{i}$ & $S_{i}(z)$ \\
\hline \hline 1 & $5+2 \nu$ & $z^{5} J_{\nu}^{2}(z)$ \\
\hline 2 & 5 & $z^{5} J_{\nu}(z) J_{-\nu}(z)$ \\
\hline 3 & $5-2 \nu$ & $z^{5} J_{-\nu}^{2}(z)$ \\
\hline 4 & $3+2 \nu$ & $z^{3} J_{\nu}^{2}(z)$ \\
\hline 5 & 3 & $z^{3} J_{\nu}(z) J_{-\nu}(z)$ \\
\hline 6 & $3-2 \nu$ & $z^{3} J_{-\nu}^{2}(z)$ \\
\hline 7 & $3+2 \nu$ & $z^{4} \frac{\mathrm{d}}{\mathrm{d} z} J_{\nu}^{2}(z)$ \\
\hline 8 & 3 & $z^{4} \frac{\mathrm{d}}{\mathrm{d} z}\left(J_{\nu}(z) J_{-\nu}(z)\right)$ \\
\hline 9 & $3-2 \nu$ & $z^{4} \frac{\mathrm{d}}{\mathrm{d} z} J_{-\nu}^{2}(z)$ \\
\hline 10 & $3+2 \nu$ & $z^{5}\left(\frac{\mathrm{d}}{\mathrm{d} z} J_{\nu}(z)\right)^{2}$ \\
\hline 11 & 3 & $z^{5}\left(\frac{\mathrm{d}}{\mathrm{d} z} J_{\nu}(z)\right)\left(\frac{\mathrm{d}}{\mathrm{d} z} J_{-\nu}(z)\right)$ \\
\hline 12 & $3-2 \nu$ & $z^{5}\left(\frac{\mathrm{d}}{\mathrm{d} z} J_{-\nu}(z)\right)^{2}$ \\
\hline
\end{tabular}

Table 1

We are interested in the behavior of the finite state dependent terms, $\left\langle T_{a b}\right\rangle_{S D}$, in the limit $\eta \rightarrow 0^{-}$. To investigate this limit in detail it is useful to break up the integral in Eq. (3.22) into the three parts

$$
\left\langle T_{a b}\right\rangle_{S D}=\frac{1}{4 \pi^{2}} \int_{0}^{\lambda} \mathrm{d} k I_{a b}(k, \eta)+\frac{1}{4 \pi^{2}} \int_{\lambda}^{-Z / \eta} \mathrm{d} k I_{a b}(k, \eta)+\frac{1}{4 \pi^{2}} \int_{-Z / \eta}^{\infty} \mathrm{d} k I_{a b}(k, \eta) .
$$

Here $\lambda$ is a finite positive constant. For $k>\lambda$ we can make use of the UV conditions (3.9), (3.10), and (3.11) in the second and third integrals. Thus, the most infrared sensitive integral is the first one. The positive constant $Z$ is 
arbitrary, provided only that $Z>-\lambda \eta$, which is always satisfied for fixed $\lambda$ and small enough $-\eta$. Hence the second integral provides the bulk of the contribution of the state dependent wave numbers that have redshifted outside the de Sitter horizon at late times. The third integral is the contribution of the state dependent terms still within the horizon which go to zero very rapidly at late times due to the UV conditions. If the $\eta \rightarrow 0^{-}$limit is uniform then all three integrals should vanish unconditionally in this limit, i.e. without any restrictions on the arbitrary parameters $\lambda$ and $Z$.

We begin by analyzing the first integral in (3.27). We are considering only fourth order adiabatic states that are IR admissible. Hence the $k$ integration converges at its lower limit and we can expand the integrand for this first integral in a series of the form (3.26) and interchange the order of summation and integration. Each term in the resulting sums contains an integral over $k$ that is finite by assumption and a factor of $(-\eta)^{2 p+\beta_{i}}$ where $p=0,1,2 \ldots$ is a non-negative integer. In fact, since at late times, $-\lambda \eta \ll 1$ for any finite $\lambda$, it is sufficient to consider only the leading $p=0$ term. From Table 1 it is clear that for $\nu<\frac{3}{2}, \beta_{i}>0$ for all $i$. Hence in the limit $\eta \rightarrow 0^{-}$, the integrand vanishes and therefore, the first integral on the right hand side of Eq. (3.27) vanishes in this late time limit.

For the second integral in (3.27) we may utilize the expansion (3.25) again. Since the integration is between finite limits for finite $\eta$ we can exchange the order of summation over $p$ and integration over $k$. Having done so we can then bound each term in the sums by taking the absolute value of its factors. Inspection of the expressions (3.23b) and (3.24d) indicates that the result is a linear combination of terms involving integrals of the three possible forms

$$
\begin{aligned}
& \mathcal{I}_{1}=\int_{\lambda}^{-Z / \eta} \frac{\mathrm{d} k}{k}\left|c_{2}(k)\right|^{2}\left(1+2 n_{k}\right)(-k \eta)^{2 p+\beta_{i}}, \\
& \mathcal{I}_{2}=\int_{\lambda}^{-Z / \eta} \frac{\mathrm{d} k}{k}\left|c_{1}(k) c_{2}(k)\right|\left(1+2 n_{k}\right)(-k \eta)^{2 p+\beta_{i}}, \\
& \mathcal{I}_{3}=\int_{\lambda}^{-Z / \eta} \frac{\mathrm{d} k}{k} n_{k}(-k \eta)^{2 p+\beta_{i}}
\end{aligned}
$$

multiplied by constant coefficients. Because of (3.11) for any $\lambda$

$$
\begin{gathered}
|C(k)|<C, \\
N(k)<N,
\end{gathered}
$$

for some real positive numbers $C$ and $N$, depending on $\lambda$. With these bounds we can bound the values of $\left|c_{2}(k)\right|$ and $n_{k}$, and use Eq. (3.5) to bound $\left|c_{1}(k)\right|$ for all $k \geq \lambda$ as follows

$$
\begin{aligned}
\left|c_{2}(k)\right| & <\frac{C}{k^{4}} \\
1 \leq\left|c_{1}(k)\right| & =\left[1+\left|c_{2}(k)\right|^{2}\right]^{\frac{1}{2}} \leq 1+\left|c_{2}(k)\right|^{2}<1+\frac{C^{2}}{k^{8}} \leq 1+\frac{C^{2}}{\lambda^{8}} \\
n_{k} & <\frac{N}{k^{4}} \\
1+2 n_{k} & <1+\frac{2 N}{k^{4}} \leq 1+\frac{2 N}{\lambda^{4}} .
\end{aligned}
$$

Using these bounds the integrals in Eq. (3.28) can be bounded as follows

$$
\begin{aligned}
\mathcal{I}_{1} & <C^{2}\left(1+2 N / \lambda^{4}\right) \int_{\lambda}^{-Z / \eta} \mathrm{d} k k^{2 p+\beta_{i}-9}(-\eta)^{2 p+\beta_{i}} \\
& =\frac{C^{2}\left(1+2 N / \lambda^{4}\right)}{\beta_{i}+2 p-8}\left[(-\eta)^{8} Z^{2 p+\beta_{i}-8}-(-\eta)^{2 p+\beta_{i}} \lambda^{2 p+\beta_{i}-8}\right] \\
\mathcal{I}_{2} & <C\left(1+C^{2} / \lambda^{8}\right)\left(1+2 N / \lambda^{4}\right) \int_{\lambda}^{-Z / \eta} \mathrm{d} k k^{2 p+\beta_{i}-5}(-\eta)^{2 p+\beta_{i}} \\
& =\frac{C\left(1+C^{2} / \lambda^{8}\right)\left(1+2 N / \lambda^{4}\right)}{\beta_{i}+2 p-4}\left[(-\eta)^{4} Z^{2 p+\beta_{i}-4}-(-\eta)^{2 p+\beta_{i}} \lambda^{2 p+\beta_{i}-4}\right] \\
\mathcal{I}_{3} & <N \int_{\lambda}^{-Z / \eta} \mathrm{d} k k^{2 p+\beta_{i}-5}(-\eta)^{2 p+\beta_{i}} \\
& =\frac{N}{\beta_{i}+2 p-4}\left[(-\eta)^{4} Z^{2 p+\beta_{i}-4}-\lambda^{2 p+(-\eta)^{2 p+\beta_{i}} \beta_{i}-4}\right]
\end{aligned}
$$


Each of these bounds vanishes in the limit $\eta \rightarrow 0^{-}$. Therefore each of the terms appearing in the second integral in (3.27) vanishes in the late time limit for $\Re(\nu)<\frac{3}{2}$.

If $\nu=\frac{1}{2}$ some of the terms will have vanishing denominators and should be interpreted according to the limiting relation

$$
\lim _{q \rightarrow 0} \frac{\left(-\frac{Z}{\eta}\right)^{q}-\lambda^{q}}{q}=\log \left(\frac{Z}{-\eta \lambda}\right)
$$

but the appearance of these logarithms does not change the result since they are always multiplied by at least $(-\eta)^{2 p+\beta_{i}}$ which vanishes for $\Re(\nu)<\frac{3}{2}$ for $p=0,1,2 \ldots$. The cases $\nu=0,1$ also involve logarithms in the Bessel function expansions but the result that the second integral in (3.27) vanishes in the late time limit is unchanged. One may consider also the case when $\nu=i \gamma$ is pure imaginary, where the forms of the coefficients (3.24d) and bilinears $S_{i}(z)$ in the table change somewhat, with again the same result. For completeness these cases are treated in detail in Appendix A.

Finally, for the third integral on the right hand side of Eq. (3.27) we do not expand the Bessel functions in powers of $(-k \eta)$. Instead we change the integration variable to $z=-k \eta$ and find integrals of the forms

$$
\begin{aligned}
& \mathcal{J}_{1}=(-\eta)^{8} \int_{Z}^{\infty} \frac{\mathrm{d} z}{z^{9}}\left|C\left(\frac{z}{-\eta}\right)\right|^{2}\left[1+2\left(\frac{-\eta}{z}\right)^{4} N\left(\frac{z}{-\eta}\right)\right] S_{i}(z) \\
& \mathcal{J}_{2}=(-\eta)^{4} \int_{Z}^{\infty} \frac{\mathrm{d} z}{z^{5}}(\Re \text { or } \Im)\left\{c_{1}\left(\frac{z}{-\eta}\right)\left[C^{*}\left(\frac{z}{-\eta}\right)\right]\right\}\left[1+2\left(\frac{-\eta}{z}\right)^{4} N\left(\frac{z}{-\eta}\right)\right] S_{i}(z), \\
& \mathcal{J}_{3}=(-\eta)^{4} \int_{Z}^{\infty} \frac{\mathrm{d} z}{z^{5}} N\left(\frac{z}{-\eta}\right) S_{i}(z)
\end{aligned}
$$

Since we are considering fourth order adiabatic states these integrals are finite for all $-\infty<\eta \leq 0$. Furthermore, the integrands of these integrals are finite throughout the entire integration range for $-\infty<\eta \leq 0$. With the lower limit $Z>0$ fixed, it is clear that we can evaluate the integrals at $\eta=0$ by first taking the limit $\eta \rightarrow 0^{-}$of the integrands and then computing the integrals. Since the integrands all vanish in the limit $\eta \rightarrow 0^{-}$, the integrals do as well. Then the third integral in (3.27) vanishes in the late time limit for $\Re(\nu)<\frac{3}{2}$. From the adiabatic four conditions (3.11) these UV contributions go to zero faster than $(-\eta)^{4}$ for any fourth order adiabatic state.

Therefore we have proven that for $\Re(\nu)<\frac{3}{2}$ all the integrals in Eq. (3.27) vanish in the limit $\eta \rightarrow 0^{-}$for an arbitrary state that is both infrared and ultraviolet finite. The fact that all three integrals vanish at late times, independently of the parameters $\lambda$ and $Z$ which we introduced to control the IR and UV contributions of the mode integral verifies that the total mode integral does converge uniformly in $\eta$, as expected. Hence all the state dependent contributions vanish at late times, $\eta \rightarrow 0^{-}$, and we have proven that in this limit $\left\langle T_{a b}\right\rangle_{r e n} \rightarrow\left\langle T_{a b}\right\rangle_{B D}$. Thus, we conclude that for $\Re(\nu)<\frac{3}{2}$ the value of the energy-momentum tensor in any physically admissable homogeneous and isotropic RW quantum state asymptotically approaches the Bunch-Davies value in de Sitter space at late times.

Moreover, from inspection of (3.31) we observe that the contribution from the upper limit at $Z$ in the second integral always falls faster than $(-\eta)^{4}$, while the contribution from the lower limit at $\lambda$ falls off only as $(-\eta)^{\beta_{i}}$ as $\eta \rightarrow 0^{-}$. This must be the same order as the first IR integral so that the arbitrary parameter $\lambda$ drops out of the final result. Hence our detailed evaluation has verified that the leading order state dependent corrections to the BD expectation value at late times come from the terms in $\left\langle T_{a b}\right\rangle_{\text {ren }}$ with the smallest $\beta_{i}$. From Table 1 these are the $i=6,9$, and 12 terms. Collecting these terms from (3.23b) and (3.24c), and the numerical coefficients from the Bessel function product formula (3.25), we conclude that the leading order behavior of the energy density and trace at late times are given by

$$
\begin{aligned}
& \varepsilon \rightarrow a^{2 \nu-3}\left[\left(\nu-\frac{1}{2}\right)^{2}+m^{2}+2(6 \xi-1)(\nu-1)\right] \int_{0}^{\infty} \mathrm{d} k k^{2-2 \nu} R(k), \quad \text { and } \\
& T \rightarrow 2 a^{2 \nu-3}\left\{-m^{2}+(6 \xi-1)\left[-\left(\nu-\frac{1}{2}\right)^{2}+2 \nu+m^{2}+2(6 \xi-1)\right]\right\} \int_{0}^{\infty} \mathrm{d} k k^{2-2 \nu} R(k),
\end{aligned}
$$

respectively, with

$$
R(k)=\frac{2^{2 \nu-3} \csc ^{2}(\pi \nu)}{\pi[\Gamma(-\nu+1)]^{2}}\left\{\left(1+2 n_{k}\right)\left[\left|c_{2}(k)\right|^{2}-\Re\left(c_{1} c_{2}^{*}\right)\right]+n_{k}\right\}
$$


The leading order state dependent contribution at late times is $a^{2 \nu-3}$ from the IR part of the mode integral, which falls off very slowly if $\Re(\nu) \rightarrow \frac{3}{2}$. We examine this latter limit in detail in the next Section.

\section{THE ALLEN-FOLACCI ATTRACTOR FOR $\Re(\nu) \rightarrow \frac{3}{2}$}

In the analysis of the previous Section we saw that the terms with the slowest falloff at late times were those with the smallest $\beta_{i}=3-2 \nu$ which behave like $a^{2 \nu-3}$ for $\nu$ real and positive, and the coefficient of this falloff is controlled by the finite $k$, IR part of the mode integral. To examine the limit $\nu \rightarrow \frac{3}{2}$ carefully, it is easiest to work with closed spatial sections and a discrete set of mode functions in order to treat the most infrared sensitive, spatially homogeneous $k=1$ mode separately from the rest, instead of dealing with an infrared sensitive continuous mode integral.

The scale factor for $\kappa=+1$ is given by Eq. (3.2) and under the variable substitution $\zeta=i \tan \eta$ the mode equation (2.6) becomes Legendre's differential equation. Hence the general solutions may be expressed in terms of associated Legendre functions $P_{-\frac{1}{2}+\nu}^{ \pm k}(\zeta)$. Since as conventionally defined these functions have a cut discontinuity on the real axis from -1 to 1 if $k$ is an odd integer, we write the fundamental complex valued solution for real $\nu$ in the form

$$
\begin{aligned}
f_{k}(\eta) & =\left[\frac{\Gamma\left(k+\frac{1}{2}+\nu\right) \Gamma\left(k+\frac{1}{2}-\nu\right)}{2}\right]^{\frac{1}{2}} \exp \left(-\frac{i k \pi}{2} \epsilon(\eta)\right) P_{-\frac{1}{2}+\nu}^{-k}(i \tan \eta) \\
& =\left[\frac{\Gamma\left(k+\frac{1}{2}+\nu\right) \Gamma\left(k+\frac{1}{2}-\nu\right)}{2}\right]^{\frac{1}{2}} \frac{e^{-i k \eta}}{k !} F\left(\frac{1}{2}+\nu, \frac{1}{2}-\nu ; k+1 ; \frac{1-i \tan \eta}{2}\right),
\end{aligned}
$$

where $\epsilon(\eta)=\theta(\eta)-\theta(-\eta)= \pm 1$ is the sign function and $F$ is the hypergeometric function. The phase factor depending on the sign of $\eta$ for odd $k$ removes the discontinuity in the $P_{-\frac{1}{2}+\nu}^{-k}$ function as $\eta$ approaches zero from positive or negative values, as the second form of (4.1) makes clear, since the $F$ function is an analytic function of $\frac{1-\zeta}{2}$, with no branch cuts for $\zeta$ on the imaginary axis. With the normalization factors chosen as in (4.1) the Wronskian condition

$$
f_{k} f_{k}^{* \prime}-f_{k}^{*} f_{k}^{\prime}=i
$$

is satisfied. Hence the general solution of the mode equation (2.6) is

$$
\psi_{k}(\eta)=\alpha_{k} f_{k}(\eta)+\beta_{k} f_{k}^{*}(\eta),
$$

with

$$
\left|\alpha_{k}\right|^{2}-\left|\beta_{k}\right|^{2}=1
$$

The Bunch-Davies state is given by $\alpha_{k}=1$ and $\beta_{k}=0$.

Now as $\nu \rightarrow \frac{3}{2}$ inspection of (4.1) shows that all the $f_{k}$ for $k>1$ are regular. In fact, in that limit the hypergeometric series for $F$ terminates and the $f_{k}(\eta)$ become the elementary functions

$$
\lim _{\nu \rightarrow \frac{3}{2}} f_{k}(\eta)=\frac{e^{-i k \eta}}{\left[2 k\left(k^{2}-1\right)\right]^{\frac{1}{2}}}(k+i \tan \eta), \quad k=2,3, \ldots,
$$

so they can be treated in the Bunch-Davies state $\alpha_{k}=1, \beta_{k}=0$ with no difficulty. However in this limit the $k=1$ mode function is singular and must be treated separately. The behavior of the $k=1$ mode as $\nu \rightarrow \frac{3}{2}$ is similar to that of a simple harmonic oscillator mode as its frequency goes to zero, i.e. in the limit where the harmonic oscillator becomes a free particle. The zero frequency limit in this simple flat space analogy is reviewed in Appendix B. Just as in that case, one can construct regular solutions to the mode equation in the limit $\nu \rightarrow \frac{3}{2}$ by taking suitable linear combinations of $f_{1}$ and $f_{1}^{*}$. In fact, the limiting form of $f_{1}(\eta)$ can be found from Sec. 2.3.1 of Ref. [35], which gives

$$
f_{1}(\eta) \rightarrow \frac{\sec \eta}{2 \sqrt{\frac{3}{2}-\nu}}-\frac{i}{2} \sqrt{\frac{3}{2}-\nu} \sec \eta(\eta+\sin \eta \cos \eta)+\ldots .
$$

We have neglected terms in the real part of $f_{1}$ that are of order $\sqrt{\frac{3}{2}-\nu}$. We have also neglected all terms that go to zero faster than this as $\nu \rightarrow \frac{3}{2}$. Extracting the scale factor $a(\eta)=\sec \eta$ we now define the real functions $u$ and $v$ by 


$$
\begin{aligned}
& u(\eta) \equiv \frac{1}{a(\eta)} \lim _{\nu \rightarrow \frac{3}{2}}\left\{\sqrt{\frac{3}{2}-\nu}\left(f+f^{*}\right)\right\}=1, \\
& v(\eta) \equiv \frac{i}{a(\eta)} \lim _{\nu \rightarrow \frac{3}{2}}\left\{\frac{1}{2 \sqrt{\frac{3}{2}-\nu}}\left(f-f^{*}\right)\right\}=\frac{\eta+\sin \eta \cos \eta}{2} .
\end{aligned}
$$

We next define new coefficients

$$
\begin{aligned}
& A \equiv-i \lim _{\nu \rightarrow \frac{3}{2}}\left\{\sqrt{\frac{3}{2}-\nu}\left(\alpha_{1}-\beta_{1}\right)\right\}, \\
& B \equiv \lim _{\nu \rightarrow \frac{3}{2}}\left\{\frac{1}{2 \sqrt{\frac{3}{2}-\nu}}\left(\alpha_{1}+\beta_{1}\right)\right\},
\end{aligned}
$$

which are finite in this limit. With these definitions the normalization is such that

$$
A^{*} B-B^{*} A=i
$$

and the limit of the general $k=1$ mode function may be written

$$
\lim _{\nu \rightarrow \frac{3}{2}} \psi_{1}(\eta)=\sec \eta(A v+B u)=a(\eta)\left[\frac{A}{2}(\eta+\sin \eta \cos \eta)+B\right] .
$$

One can also define the time-independent Hermitian operators,

$$
\begin{aligned}
Q & \equiv \frac{1}{2 \sqrt{\frac{3}{2}-\nu}}\left[\left(\alpha_{1}+\beta_{1}\right) a_{1}+\left(\alpha_{1}+\beta_{1}\right)^{*} a_{1}^{\dagger}\right] \rightarrow B a_{1}+B^{*} a_{1}^{\dagger}, \\
P & \equiv-i \sqrt{\frac{3}{2}-\nu}\left[\left(\alpha_{1}-\beta_{1}\right) a_{1}-\left(\alpha_{1}-\beta_{1}\right)^{*} a_{1}^{\dagger}\right] \rightarrow A a_{1}+A^{*} a_{1}^{\dagger},
\end{aligned}
$$

obeying the canonical commutation relations, $[Q, P]=i$. They also remain finite in the limit $\nu \rightarrow \frac{3}{2}$.

We will consider the limit $\nu \rightarrow \frac{3}{2}$ of the energy density in the particular order of fixing the mass of the scalar field to be $m=0$ and letting the dimensionless parameter $\xi$ approach zero, since this case is relevant for the infrared scaling analysis of Section VI. From (3.4) with $m=0$ and $\xi$ small we have

$$
\xi \simeq \frac{(3-2 \nu)}{8} \rightarrow 0
$$

The complementary case, $\xi=0$ and $m^{2} \rightarrow 0$ is similar and has been discussed in Refs. [17] and [19]. From (2.9a) we read the energy density in the $k=1$ mode for $m=0$

$$
\varepsilon_{1}=-\left\langle T^{0}{ }_{0}\right\rangle_{k=1}=\frac{\left(1+2 n_{1}\right)}{4 \pi^{2} a^{4}}\left\{\left|\psi_{1}^{\prime}\right|^{2}+\left|\psi_{1}\right|^{2}+(6 \xi-1)\left[\tan \eta\left(\psi_{1} \psi_{1}^{* \prime}+\psi_{1}^{*} \psi_{1}^{\prime}\right)-\left(\tan ^{2} \eta-1\right)\left|\psi_{1}\right|^{2}\right]\right\} .
$$

We are interested first in the asymptotic form of this energy density at late times, $\eta \rightarrow \frac{\pi}{2}$, and then in the limiting form of the resulting expression as $\xi \rightarrow 0$ according to (4.12). The asymptotic late time limit of the mode function $f_{1}(\eta)$ for any $\nu>0$ can be found from the inversion transformation of the hypergeometric function, given by formula 2.1.4 (17) of 35. We find that

$$
\begin{aligned}
f_{1}\left(\eta \rightarrow \frac{\pi}{2}\right) & \rightarrow\left[\frac{\Gamma\left(\frac{3}{2}-\nu\right)}{2 \Gamma\left(\frac{3}{2}+\nu\right)}\right]^{\frac{1}{2}} \frac{\Gamma(2 \nu)}{\Gamma\left(\frac{1}{2}+\nu\right)}(-i)\left(\frac{i \sec \eta}{2}\right)^{\nu-\frac{1}{2}} \sim a^{\nu-\frac{1}{2}} \\
f_{1}^{\prime} & \rightarrow\left(\nu-\frac{1}{2}\right) \sec \eta f_{1} \sim a^{\nu+\frac{1}{2}}
\end{aligned}
$$

as $\eta \rightarrow \frac{\pi}{2}$, which is the same late time behavior in terms of the scale factor that we found in the spatially flat coordinates. Notice that at $\nu=\frac{3}{2}$ the phase factor cancels and these limiting forms of the oscillatory mode functions 
become real, while the $\Gamma$ function has a pole singularity there. Thus, keeping only the leading behavior as $\nu$ approaches $\frac{3}{2}$ we find that the late time limits of the mode function and its derivative are

$$
\begin{aligned}
& \psi_{1}\left(\eta \rightarrow \frac{\pi}{2}\right) \rightarrow \frac{\alpha_{1}+\beta_{1}}{2 \sqrt{\frac{3}{2}-\nu}} a^{\nu-\frac{1}{2}}, \\
& \psi_{1}^{\prime}\left(\eta \rightarrow \frac{\pi}{2}\right) \rightarrow \frac{\alpha_{1}+\beta_{1}}{2 \sqrt{\frac{3}{2}-\nu}} a^{\nu+\frac{1}{2}} .
\end{aligned}
$$

Thus, the dominant terms in the $k=1$ energy density $\varepsilon_{1}$ in this limit are $\left|\psi_{1}^{\prime}\right|^{2}$ and the terms involving $\frac{a^{\prime}}{a}=\tan \eta$, and we obtain

$$
\lim _{\eta \rightarrow \frac{\pi}{2}} \varepsilon_{1}=\left[\frac{3\left(1+2 n_{1}\right)}{32 \pi^{2}}\left|\alpha_{1}+\beta_{1}\right|^{2}+\mathcal{O}(3-2 \nu)\right] a^{2 \nu-3}
$$

The singularity in the $\Gamma$ function has canceled against the $\xi$ in the numerator. The remaining coefficient is clearly state dependent. From this asymptotic form of the energy density in the $k=1$ mode at late times it is clear that for $\nu$ close to but still less than $\frac{3}{2}$, the state-dependent energy density $\varepsilon_{1}$ goes to zero at late times, albeit very slowly, which is consistent with our previous flat section analysis.

The corresponding expression for the trace in the $k=1$ mode is

$$
-\varepsilon_{1}+3 p_{1}=\frac{\left(1+2 n_{1}\right)}{2 \pi^{2} a^{4}}(6 \xi-1)\left\{-\left|\psi_{1}^{\prime}\right|^{2}+\left[\tan \eta\left(\psi_{1} \psi_{1}^{* \prime}+\psi_{1}^{*} \psi_{1}^{\prime}\right)+\left[\sec ^{2} \eta+2(6 \xi-1) \sec ^{2} \eta\right]\left|\psi_{1}\right|^{2}\right]\right\} .
$$

Substituting the late time asymptotic forms (4.16) to leading order in $\xi$ as before yields

$$
-\varepsilon_{1}+3 p_{1} \rightarrow\left[-\frac{3\left(1+2 n_{1}\right)}{8 \pi^{2}}\left|\alpha_{1}+\beta_{1}\right|^{2}+\mathcal{O}(3-2 \nu)\right] a^{2 \nu-3} \rightarrow-4 \varepsilon_{1} .
$$

Hence, $p_{1} \rightarrow-\varepsilon_{1}$ and the contribution from this mode is de Sitter invariant at late times for any initial physical state.

Since the Bunch-Davies state is $\alpha_{k}=1$ and $\beta_{k}=n_{k}=0$ for all $k$ and this state has a renormalized $\left\langle T_{a b}\right\rangle$ which is strictly time independent for all $\nu$, the time dependent contribution in (4.17) and (4.19) for $\nu<\frac{3}{2}$ in the $k=1$ mode must be canceled by a time dependent contribution from all the other modes in the renormalized energy-momentum tensor. In other words, the late time behavior of all the $k>1$ modes in the BD state must be

$$
\left.\varepsilon_{B D}\right|_{k>1} \rightarrow \varepsilon_{B D}-\left[\frac{3}{32 \pi^{2}}+\mathcal{O}(3-2 \nu)\right] a^{2 \nu-3}
$$

for $\nu$ close to $\frac{3}{2}$. The pressure for the $k>1$ modes is obtained from this by the de Sitter invariant relation $p=-\varepsilon$.

The subtraction of the second term in $(4.20)$ can be understood in a different way. Consider the short distance expansion of the BD two-point function for $\nu<\frac{3}{2}$, namely [19]

$$
G_{B D}\left(x, x^{\prime}\right) \rightarrow \frac{1}{8 \pi^{2}}\left[\frac{1}{1-Z}-\log (1-Z)+\frac{1}{\frac{3}{2}-\nu}\right]
$$

for the de Sitter invariant bi-scalar, $Z\left(x, x^{\prime}\right) \rightarrow 1$ and $\nu$ close to $\frac{3}{2}$. The constant term is singular at $\nu=\frac{3}{2}$, but it gives a finite contribution to the energy density from the $\xi G_{a b} \phi^{2}$ term in the energy-momentum tensor. In the dimensionless units we are using this is equal to

$$
\frac{3}{8 \pi^{2}} \xi\left(\frac{R}{12}\right)^{2} \frac{1}{\frac{3}{2}-\nu}=\frac{3}{32 \pi^{2}},
$$

for $\xi \rightarrow 0$ according to (4.12). The last constant term in (4.21) is absent in the short distance expansion of the Allen-Folacci two-point function [17], and in the Bunch-Davies two-point function it comes entirely from the $k=1$ mode in the BD state. Hence the contribution to the energy density of the $k>1$ modes in the BD state does not contain (4.22), and must equal the full $\varepsilon_{B D}$ minus (4.22) from the $k=1$ mode, which is equivalent to (4.20) at $\nu=\frac{3}{2}$.

With the $k=1$ and $k>1$ mode contributions separated we are now in a position to take the limit of $\nu \rightarrow \frac{3}{2}$. Apparently we would obtain a state dependent contribution from (4.17). However, inspection of (4.10) shows that 
requiring the $k=1$ mode function to be regular as $\nu \rightarrow \frac{3}{2}$ is equivalent to requiring that $A$ and $B$ remain finite in this limit. From (4.8) we then have

$$
\alpha_{1}+\beta_{1} \rightarrow 2 B \sqrt{\frac{3}{2}-\nu} \rightarrow 0 .
$$

Therefore the apparently state dependent contribution (4.17) from the $k=1$ mode vanishes in any infrared finite state parameterized by finite $A$ and $B$, while the $k>1$ contribution is still given by (4.20), and we have the result

$$
\varepsilon_{R}=\left.\lim _{\xi \rightarrow 0} \varepsilon_{B D}(\xi)\right|_{m=0}-\frac{3}{32 \pi^{2}}\left(\frac{R}{12}\right)^{2}=\varepsilon_{A F},
$$

in any $(A, B)$ "vacuum" state with $\alpha_{k}=1, \beta_{k}=n_{k}=0$ for $k>1$, upon restoring physical units. The difference here is just that required to give the Allen-Folacci $(\mathrm{AF})$ renormalized expectation value, provided the limit of the $\mathrm{BD}$ value is taken in the same order of $m=0, \xi \rightarrow 0$ that we have evaluated (4.17). Since from (3.6)

$$
\left.\varepsilon_{B D}(\xi)\right|_{m=0}=\frac{3}{16 \pi^{2}}\left(\frac{R}{12}\right)^{2}\left[\frac{1}{180}-\frac{1}{6}(6 \xi-1)^{2}\right]
$$

and both the $\mathrm{BD}$ and $\mathrm{AF}$ values are de Sitter invariant,

$$
\begin{aligned}
\left.\lim _{\xi \rightarrow 0}\left\langle T_{a b}\right\rangle_{r e n}(\xi)\right|_{m=0} & =-\frac{3 g_{a b}}{16 \pi^{2}}\left(\frac{R}{12}\right)^{2}\left(\frac{1}{180}-\frac{1}{6}-\frac{1}{2}\right) \\
& =\frac{119}{960 \pi^{2}}\left(\frac{R}{12}\right)^{2} g_{a b}=\frac{119 R^{2}}{138240 \pi^{2}} g_{a b}=\left\langle T_{a b}\right\rangle_{A F},
\end{aligned}
$$

and we obtain the de Sitter invariant AF result for all $(A, B)$ "vacuum" states in the massless, minimally coupled case.

With the above careful analysis of the spatially homogeneous mode and the independence of the asymptotic value of its energy-momentum tensor in an arbitrary $k=1$ infrared finite state characterized by $A$ and $B$, it is now straightforward to carry out the proof of the attractor behavior of the AF state for an arbitrary UV finite physical state with $m=\xi=0$, by allowing the $k>1$ modes to have $\beta_{k}$ and $n_{k}$ different from zero. Substituting (4.3), (4.5), (4.10), and (4.16) into (2.9a), 2.9b) with $m=\xi=0$, and using (4.4) gives

$$
\begin{aligned}
\varepsilon=-\left\langle T^{0}{ }_{0}\right\rangle_{\text {ren }}= & -\left\langle T^{0}{ }_{0}\right\rangle_{A F}+\left(1+2 n_{1}\right) \frac{|A|^{2} \cos ^{6} \eta}{\pi^{2}} \\
& +\frac{1}{4 \pi^{2}} \sum_{k=2}^{\infty}\left\{\left[2 n_{k}+2\left(1+2 n_{k}\right)\left|\beta_{k}\right|^{2}\right]\left[k^{3} \cos ^{4} \eta+k\left(-\cos ^{4} \eta+\frac{1}{2} \cos ^{2} \eta\right)\right]\right. \\
& +\left(1+2 n_{k}\right)\left[\left(\beta_{k} \alpha_{k}^{*} e^{2 i k \eta}+\beta_{k}^{*} \alpha_{k} e^{-2 i k \eta}\right) k\left(-\cos ^{4} \eta+\frac{1}{2} \cos ^{2} \eta\right)\right. \\
& \left.\left.+i\left(\beta_{k} \alpha_{k}^{*} e^{2 i k \eta}-\beta_{k}^{*} \alpha_{k} e^{-2 i k \eta}\right) k^{2} \cos ^{3} \eta \sin \eta\right]\right\} \\
\langle T\rangle_{r e n}= & \langle T\rangle_{A F}+\left(1+2 n_{1}\right) \frac{2|A|^{2} \cos ^{6} \eta}{\pi^{2}} \\
& -\frac{1}{4 \pi^{2}} \sum_{k=2}^{\infty}\left\{\left[2 n_{k}+2\left(1+2 n_{k}\right)\left|\beta_{k}\right|^{2}\right] k \cos ^{2} \eta\right. \\
& +\left(1+2 n_{k}\right)\left[\left(\beta_{k} \alpha_{k}^{*} e^{2 i k \eta}+\beta_{k}^{*} \alpha_{k} e^{-2 i k \eta}\right)\left(-2 k^{3} \cos ^{4} \eta+k \cos ^{2} \eta\right)\right. \\
& \left.\left.+2 i\left(\beta_{k} \alpha_{k}^{*} e^{2 i k \eta}-\beta_{k}^{*} \alpha_{k} e^{-2 i k \eta}\right) k^{2} \cos ^{3} \eta \sin \eta\right]\right\} .
\end{aligned}
$$

Provided the $k$ sums converge, it is clear that all the state dependent terms contain at least one factor of $a^{-2}=\cos ^{2} \eta$, and so vanish in the limit of $\eta \rightarrow \frac{\pi}{2}$. However, the requirement that the state be fourth order adiabatic just guarantees this convergence, for the same reason as in the previous analysis in spatially flat coordinates. Indeed we have

$$
\begin{gathered}
\left|\beta_{k}\right|=\frac{C(k)}{k^{4}}, \\
n_{k}=\frac{N(k)}{k^{4}},
\end{gathered}
$$


for some $C(k)$ and $N(k)$ that vanish as $k \rightarrow \infty$. This is sufficient to guarantee the absolute convergence of all terms in the sums. Since all state dependent terms are multiplied by at least two powers of $\cos \eta=a^{-1}$, which vanishes in the late time limit $\eta \rightarrow \frac{\pi}{2}$, we conclude that any UV and IR admissible state of the massless, minimally coupled scalar field has an energy-momentum tensor which approaches the AF value, $\left\langle T_{a b}\right\rangle_{A F}$ in the late time limit $\eta \rightarrow \frac{\pi}{2}$.

If one considers the contributions of the state dependent terms to the energy density and trace from the $k>1$ modes, for $\nu$ not exactly $\frac{3}{2}$, the kinematics is essentially the same as our previous analysis of the $k=1$ mode. Their contribution also falls off proportional to $\xi\left|\alpha_{k}+\beta_{k}\right|^{2} a^{2 \nu-3}$ at late times for $\nu$ close to $\frac{3}{2}$. However, there is no compensating large factor coming from the pole in the $\Gamma$ function normalization constant as there is for the $k=1$ mode. Hence, the coefficient of this slow fall off goes to zero as the massless, minimally coupled limit is approached. That is, exactly at $m=\xi=0$ when the contribution of the $k>1$ modes no longer falls off and they could in principle contribute to the asymptotic value of $\left\langle T_{a b}\right\rangle_{r e n}$ at late times, at that very point their coefficient vanishes identically and they make no contribution at all. Thus, at precisely $m=0$ and $\xi=0$ the difference between the BD and AF values can be attributed entirely to the additional condensate in the spatially homogeneous $k=1$ mode alone, and there are no slow transient modes in our explicit analysis of the massless, minimally coupled energy and trace.

These considerations are relevant to the case when $\nu=\frac{3}{2}$ but $m$ and $\xi$ are separately non-zero. The calculation is almost identical but the conclusion is different, since now the finite $k>1$ mode sum is multiplied by a coefficient $\xi$ which does not vanish. The entire mode sum of state independent terms from $k=2$ up to $a(\eta)$ do not fall off at late times and in fact all add up, to give a contribution proportional to $\left\langle\Phi^{2}\right\rangle \sim \sum_{k=2}^{a} k^{-1} \sim \log a$ in $\left\langle T_{a b}\right\rangle_{\text {ren }}$, which grows linearly in comoving time. The explicit expression is most conveniently calculated by using (2.11) to divide the energy-momentum tensor into a state dependent numerical part, $\left\langle T_{a b}\right\rangle_{n}$ and a state independent analytic part, $\left\langle T_{a b}\right\rangle_{a n}$. They are separately conserved [33. The quantities $\left\langle T_{a b}\right\rangle_{d}$ and $\left\langle T_{a b}\right\rangle_{a n}$ are given in Ref. [33]. By substituting Eqs. (4.16) and (4.5) into Eqs. (2.9a) and (2.9b), and subtracting the expression for $\left\langle T_{a b}\right\rangle_{d}$ given in Ref. [33], we find that $\left\langle\overline{T_{a b}}\right\rangle_{n}$ approaches a state dependent, finite constant in the limit $\eta \rightarrow \frac{\pi}{2}$. However, the quantity $\left\langle T_{a b}\right\rangle_{a n}$ has a term that is proportional to $\left\langle\Phi^{2}\right\rangle$. At late times this term behaves as $\log a$ and dominates. In fact

$$
\begin{aligned}
\varepsilon_{\text {ren }} & \rightarrow-\frac{3 \xi}{4 \pi^{2}} \log (\cos \eta)+q_{1}, \\
\langle T\rangle_{\text {ren }} & \rightarrow \frac{3 \xi}{\pi^{2}} \log (\cos \eta)+q_{2},
\end{aligned}
$$

with the constants $q_{1}$ and $q_{2}$ dependent on the state of the field and constrained by the conservation equation. Since $\log (\cos \eta) \rightarrow t$ in comoving time, the energy-momentum tensor grows linearly with $t$ at late times. For $\xi>0$ (and $m^{2}<0$ ) the linear growth in comoving time decreases the effective cosmological "constant", whereas for $\xi<0$ (and $\left.m^{2}>0\right)$ it increases it. In either case the back-reaction of the energy-momentum of the quantum scalar field for $m^{2}+\xi R=0$ (but $m^{2}=-\xi R \neq 0$ ) on the geometry certainly cannot be neglected at late times since $\left\langle T_{a b}\right\rangle$ grows without bound for any physical state. The fact that a massive non-minimal field with $\xi<0$ can induce an effective cosmological "constant" due to inflationary particle production was noted in Ref. [36] using a different approach. It is an interesting open question whether this linearly growing behavior (in proper time) carries over to the physically more relevant case of one-loop gravitons, since the mode functions for gravitons in a particular gauge obey the same equation in a RW spacetime as do the mode functions for a massless minimally coupled scalar field 37.

Finally, we note that for all real values of $\nu$, the analysis of the $k=1$ mode in this Section may be extended to all of the higher $k$ modes, since the late time behavior of the higher $k$ modes is determined by that of the hypergeometric function in (4.1), which gives

$$
f_{k}\left(\eta \rightarrow \frac{\pi}{2}\right) \rightarrow\left[\frac{\Gamma\left(k+\frac{1}{2}-\nu\right)}{2 \Gamma\left(k+\frac{1}{2}+\nu\right)}\right]^{\frac{1}{2}} \frac{\Gamma(2 \nu)}{\Gamma\left(\frac{1}{2}+\nu\right)} \frac{(-i)^{k}}{k !}\left(\frac{i \sec \eta}{2}\right)^{\nu-\frac{1}{2}}
$$

Thus all of the higher $k$ modes behave as $a^{\nu-\frac{1}{2}}$ and give the (unrenormalized) energy density the leading order late time behavior

$$
a^{2 \nu-3}\left[\left(\nu-\frac{3}{2}\right)^{2}+12 \xi(\nu-1)+m^{2}\right]\left[\frac{\Gamma(2 \nu)}{2^{\nu} \Gamma\left(\nu+\frac{1}{2}\right)}\right]^{2} \sum_{k=1}^{\infty}\left(1+2 n_{k}\right) \frac{\Gamma\left(k+\frac{1}{2}-\nu\right)}{\Gamma\left(k+\frac{1}{2}+\nu\right)(k !)^{2}}\left|\alpha_{k}+e^{i \pi\left(k+\nu-\frac{1}{2}\right)} \beta_{k}\right|^{2},
$$

which grows (or shrinks) like $a^{2 \nu-3}$ for an arbitrary physical state unless either the coefficient vanishes or $\alpha_{k}+$ $e^{i \pi\left(k+\nu-\frac{1}{2}\right)} \beta_{k}=0$ for all $k$. However, the latter is impossible since it is inconsistent with the requirement that all physically allowable states be fourth order adiabatic states, which requires that $\alpha_{k} \rightarrow 1$ and $\beta_{k} \rightarrow 0$ sufficiently fast as $k \rightarrow \infty$, in order for the renormalized energy density to be UV finite. Since the fourth order adiabatic subtraction 
behaves at most like a constant at late times, the $a^{2 \nu-3}$ behavior is not affected by the UV subtraction, and indeed the sum in 4.31 converges at large $k$ for any UV admissable state. Hence after the adiabatic four subtraction the leading order late time behavior indicated by (4.31) survives unless the first bracket in front of the entire expression vanishes. The quantity in this bracket is identical to the factor in (3.34a) found in the flat spatial section analysis of the last Section. The corresponding quantity for the trace is given by the factor in curly brackets in (3.34b), which is a similar combination of $\nu, \xi$, and $\mathrm{m}^{2}$. If we require that both of these factors vanish identically, to eliminate the leading order behavior in all components of $\left\langle T_{a b}\right\rangle_{r e n}$, then these two conditions plus the defining relation (3.4) give

$$
\begin{aligned}
m^{2} & =-\frac{\nu(2 \nu-3)(2 \nu-1)}{4(\nu-2)}, \\
\xi & =\frac{(2 \nu-3)}{8(\nu-2)} .
\end{aligned}
$$

Thus, except for $\nu=2$, for any given $\nu$ there is always one value of $m^{2}$ and one value of $\xi$ for which the coefficients of these leading order terms in $\left\langle T_{a b}\right\rangle_{\text {ren }}$ vanish. The next to leading order terms go like $a^{2 \nu-5}$, and grow without bound at late times in any case when $\nu>\frac{5}{2}$.

The above analysis implies that, for most values of $m^{2}$ and $\xi$, when $\nu>\frac{3}{2}$ the leading order terms in the components of the energy-momentum tensor grow without bound like $a^{2 \nu-3}$ in de Sitter space for any physically admissable initial state of the scalar field. These values of $\nu$ correspond to the purely tachyonic cases $m^{2}+\xi R<0$.

\section{NUMERICAL STUDIES}

In this Section we display numerical results for various values of $\nu$. All results are given in dimensionless units where $\alpha=1$ and $R=12$. The quantum state in each case is a fourth order adiabatic state matched to the vacuum at some initial time $\eta_{0}$. That is, we choose initial conditions for the mode function and its first derivative to be

$$
\begin{aligned}
\psi_{k}\left(\eta_{0}\right) & =\psi_{k}^{(4)}\left(\eta_{0}\right), \\
\frac{\mathrm{d} \psi_{k}}{\mathrm{~d} \eta}\left(\eta_{0}\right) & =\frac{\mathrm{d} \psi_{k}^{(4)}}{\mathrm{d} \eta}\left(\eta_{0}\right),
\end{aligned}
$$

with $\psi_{k}^{(4)}$ the fourth order adiabatic mode function defined by (3.7) with phase measured from the initial time $\eta_{0}$. The initial time was chosen to be $t_{0}=1$ in comoving coordinates, i.e. $\sec \eta_{0}=\cosh (1)=1.54308 \ldots$..

For $\Re(\nu)<\frac{3}{2}$ the proof in Section III] states that the energy-momentum tensor approaches the Bunch-Davies value (3.6) at late times for an arbitrary physically admissable state. This occurs due to a redshifting of the state dependent part of the energy-momentum tensor. The initial state dependent transient contributions fall off like

$$
a^{2 \nu-3}=(\cosh t)^{2 \nu-3}
$$

Thus the characteristic time to approach the $\mathrm{BD}$ value is

$$
\tau=\frac{1}{3-2 \nu} .
$$

In Fig. 1 we plot the renormalized energy density for these adiabatic initial conditions with $m=0$ and $\xi=\frac{1}{7}$. For this relatively large value of $\xi$, the characteristic time $\tau$ is of order one and the energy density approaches the BD value within one expansion time.

For smaller values of $\xi$ the initial value transients persist for longer times. Our analysis in the previous Section shows that the $k=1$ contribution is essential for the shift from the BD to AF value as $\xi \rightarrow 0$. Since the $k=1$ mode in an arbitrary physical state contributes to the energy density the value $\varepsilon_{1}$ given by (4.17), up to terms which fall off like $a^{-2}$, for arbitrary $\alpha_{1}, \beta_{1}$, and $n_{1}$, while the BD state has $\alpha_{1}=1, \beta_{1}=n_{1}=0$, it is clear that the difference of the renormalized energy density from the $\mathrm{BD}$ value coming from this mode is

$$
\varepsilon_{R}-\varepsilon_{B D}=\varepsilon_{1}-\frac{3}{32 \pi^{2}}\left(\frac{R}{12}\right)^{2} a^{2 \nu-3} .
$$

For the adiabatic initial conditions here we find that 


$$
\psi_{1}\left(\eta_{0}\right)=\frac{1}{\sqrt{2 W_{1}^{(4)}\left(\eta_{0}\right)}} \approx \frac{\left(\alpha_{1}+\beta_{1}\right) \sec \eta_{0}}{2 \sqrt{\frac{3}{2}-\nu}}
$$

Since $W_{1}^{(4)}\left(\eta_{0}\right)$ remains finite as $\xi \rightarrow 0$, provided that $\eta_{0} \neq 0$, it follows that in this limit $\left|\alpha_{1}+\beta_{1}\right|^{2} \sim 3-2 \nu \sim \xi$ also goes to zero. Hence for small $\xi, \varepsilon_{R}$ goes to a value close to (but slightly larger than) the AF value after a time of order one. This is observed in both Figs. 2 and 3 for $\xi=\frac{1}{100}$ and $\xi=\frac{1}{1000}$, respectively.

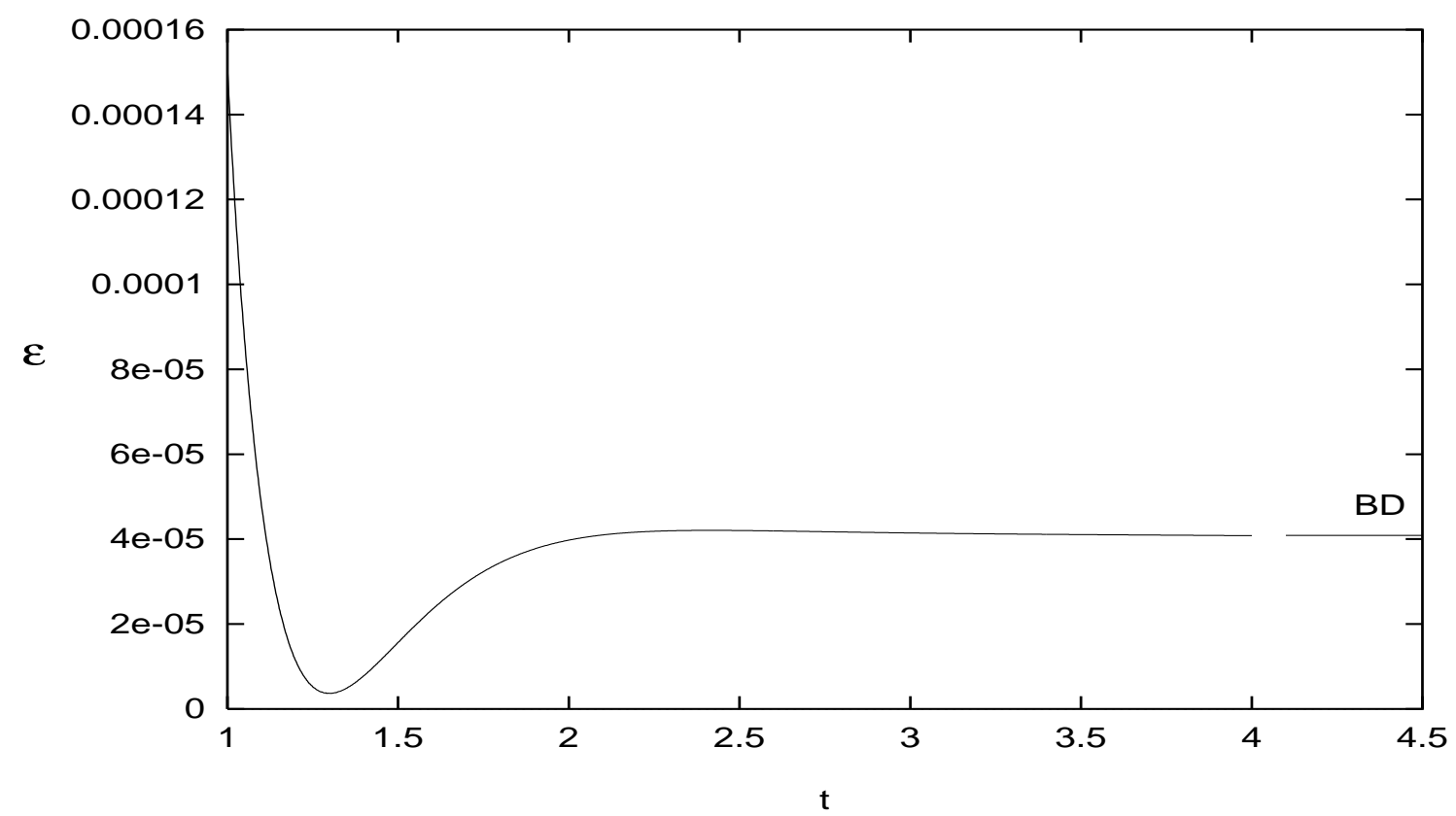

FIG. 1. The energy density for $m=0$ and $\xi=\frac{1}{7}$ as a function of comoving time $t$ for adiabatic initial conditions at $t=1$. The attractor behavior of the Bunch-Davies value for the energy density is illustrated.

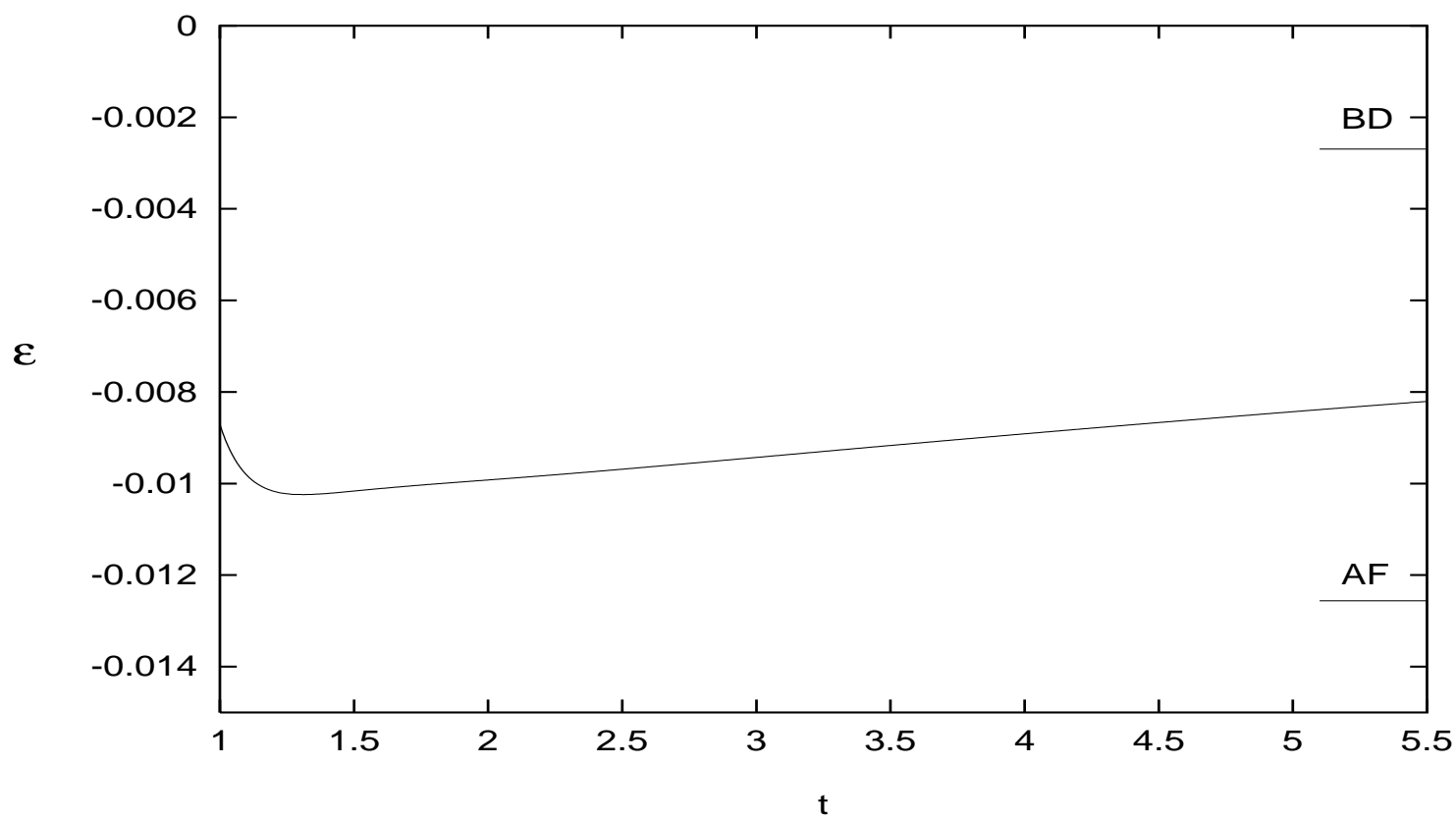

FIG. 2. The energy density for $m=0$ and $\xi=\frac{1}{100}$ as a function of comoving time $t$ for adiabatic initial conditions at $t=1$. The line segments at right giving the values of the energy density for the Allen-Folacci (AF) state, and the Bunch-Davies (BD) state (for $m=0$ and $\xi=\frac{1}{100}$ ). 


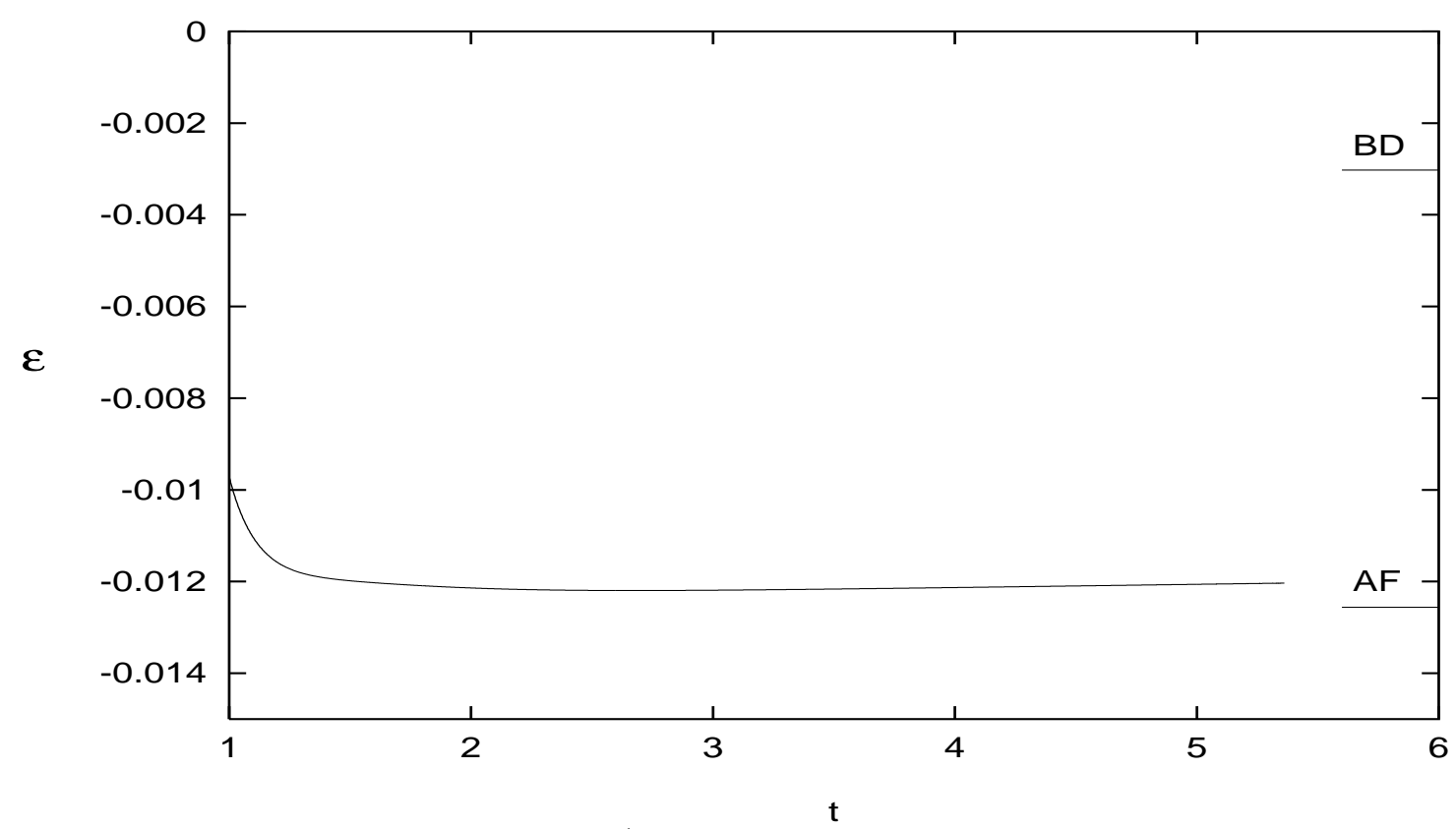

FIG. 3. The energy density for $m=0$ and $\xi=\frac{1}{1000}$ for the same initial conditions as the previous figures. Notice the very long-lived transient behavior of $\varepsilon$, staying close to the AF value with a small positive linear slope towards the $\mathrm{BD}$ value.

Further, the energy-momentum tensor contains a term proportional to $\xi\left\langle\Phi^{2}\right\rangle$ which would grow linearly in comoving time for $\nu$ very close to $\frac{3}{2}$, except for the factor of $a^{2 \nu-3}$ that damps it to zero at very late times $t \gg \tau$. Hence on times $1<t \ll \tau$, where the $a^{2 \nu-3}$ factor is essentially constant, we should expect this linear growth of $\varepsilon$ in comoving time with a slope proportional to $\xi$, given by (4.29a). This behavior is demonstrated in Figs. 2 and 3 . In the latter case $\xi$ is so small that $\alpha_{1}+\beta_{1}$ and the slope are nearly vanishing and the energy density stays close to the $\mathrm{AF}$ value until times of order $\tau=125$, which is much larger than the times shown in Fig. 3. When $\xi=0$ (still keeping $m=0$ ) both $\alpha_{1}+\beta_{1}$ and the slope vanishes identically, so the energy-momentum tensor goes to the AF value and remains there. This demonstrates that the limit $\Re(\nu) \rightarrow \frac{3}{2}$ is quite continuous when viewed at finite times with well-defined physical initial conditions, although the late time limit is discontinuous.

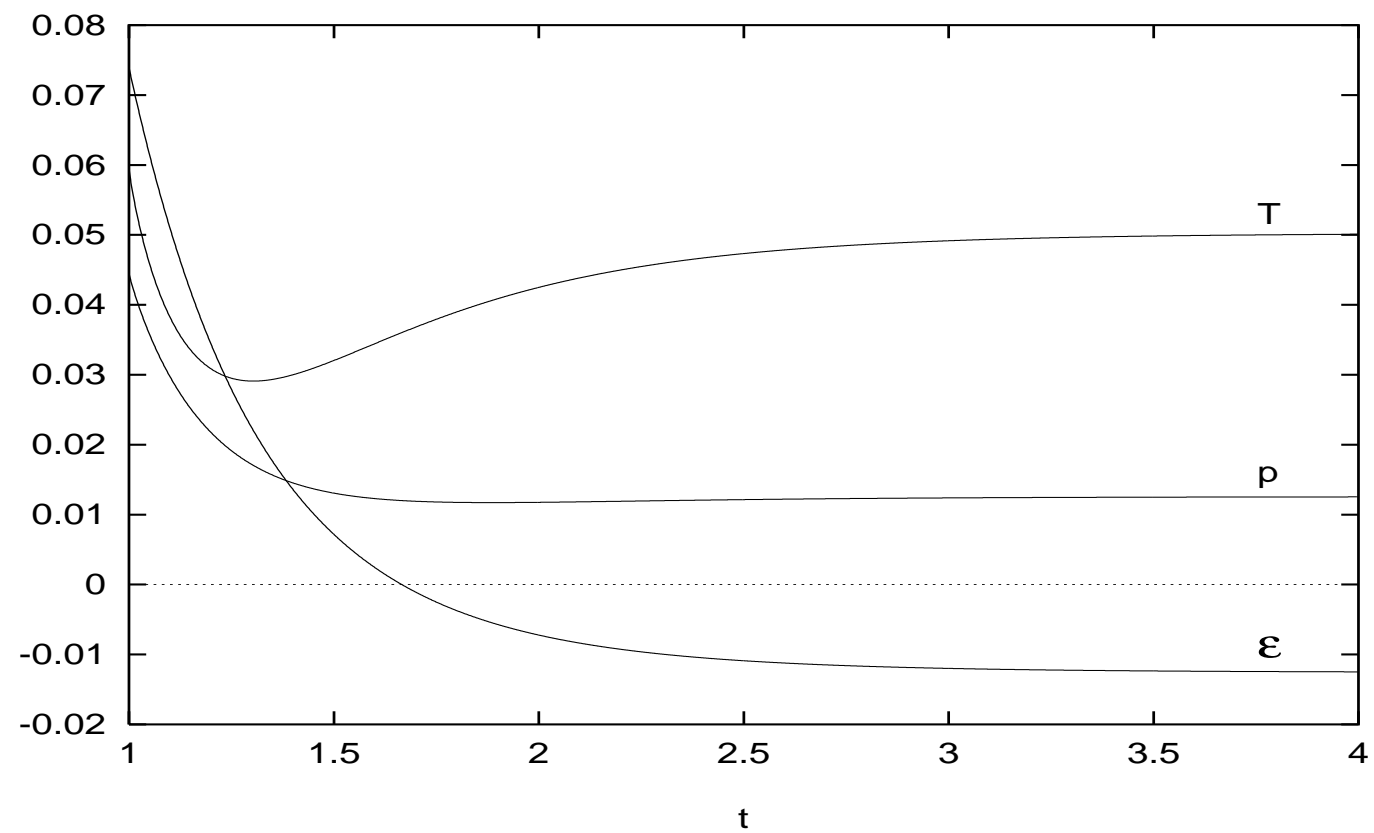

FIG. 4. From top to bottom the curves are the trace, pressure, and energy density for $m=0$ and $\xi=0$. The state is a fourth order adiabatic state at $t=1$ with $n_{1}=n_{2}=2$ and $n_{k}=0$ for all $k>2$. The trace, pressure, and energy density approach their AF fixed point values at late times. 
In the case $\nu=\frac{3}{2}$ there are two distinct behaviors depending on whether $m$ and $\xi$ are separately vanishing or not. In the massless, minimally coupled case we proved in Section IV that the Allen-Folacci value is a fixed point at late times. In Figure 4 we show the approach of the energy density, pressure, and trace to their Allen-Folacci values for the massless minimally coupled field. The field is in an "n-particle" state with $n_{1}=n_{2}=2$ and $n_{k}=0$ for all $k>2$. In Figure 5 we show the behavior of the energy density and trace for the case $m=0.3$ and $\xi=-0.0075$ when the field is in a fourth order adiabatic vacuum state.

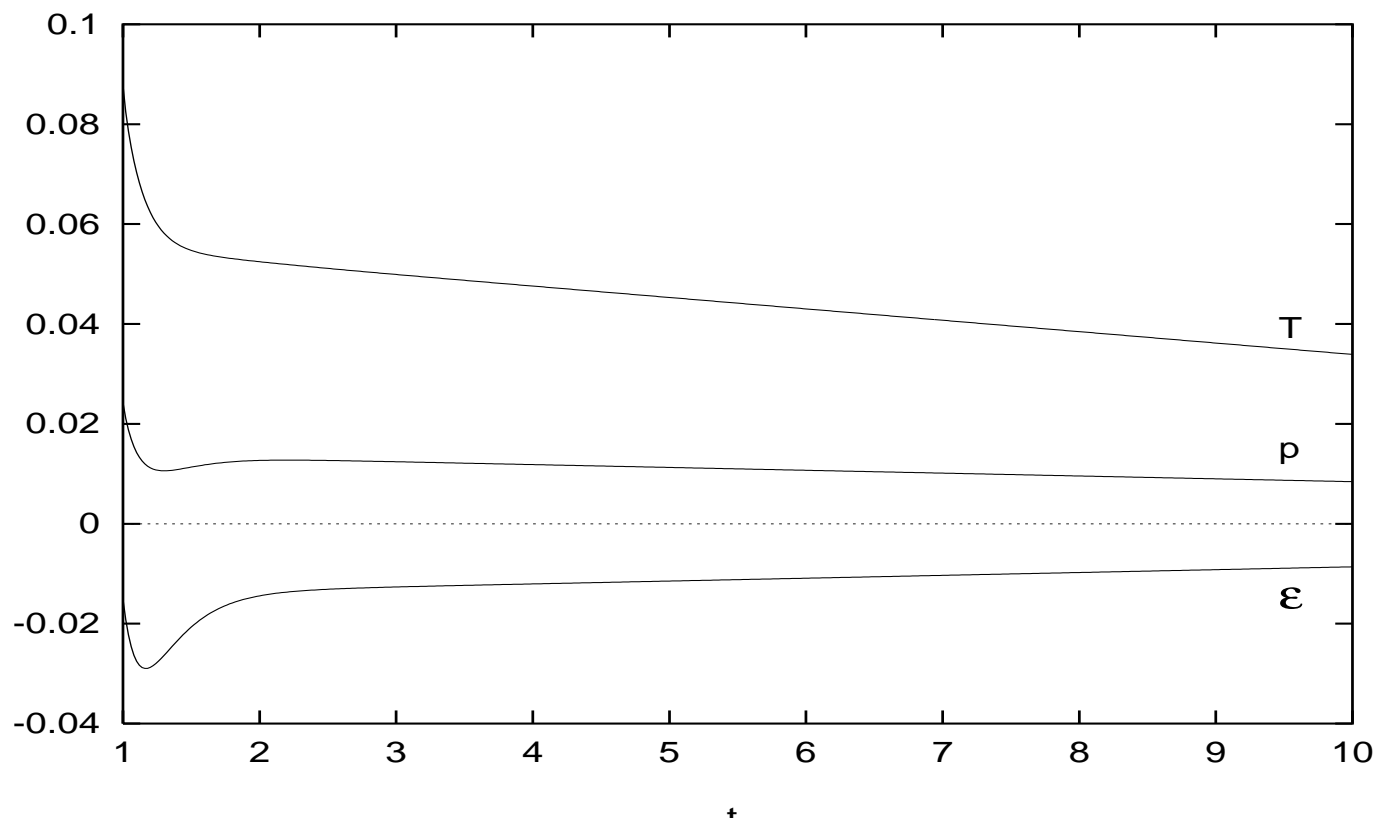

FIG. 5. From top to bottom the curves are the trace, pressure, and energy density for $m=0.3$ and $\xi=-0.0075, n_{k}=0$ and the fourth order adiabatic state at the initial time $t=1$. The curves behave linearly in comoving time $t$ at late times, with their slopes given by Eqs. (4.29a and 4.29b).

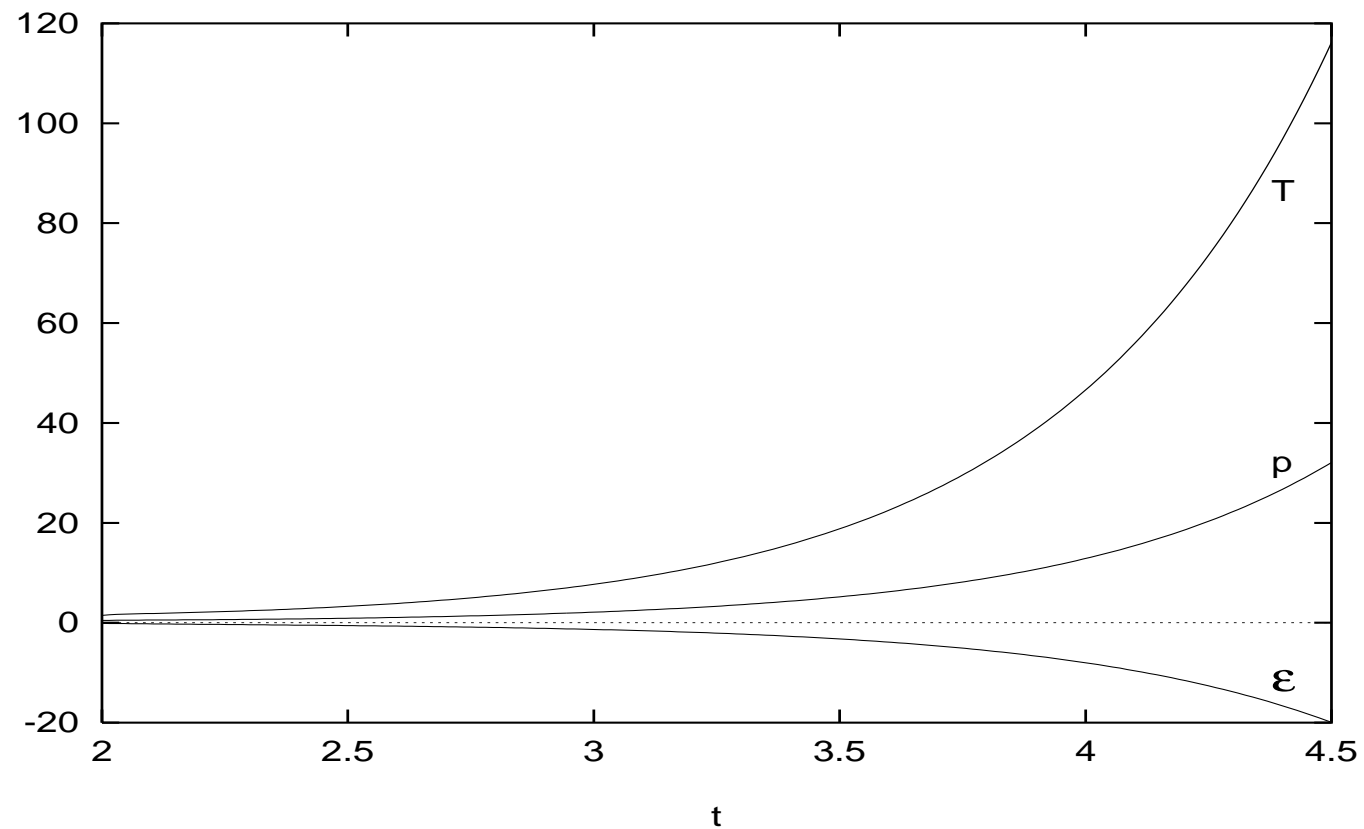

FIG. 6. From top to bottom the curves are the trace, pressure, and energy density for $m=0.1$ and $\xi=-0.3, n_{k}=0$ and the fourth order adiabatic state at the initial time $t=1$.

When $\Re(\nu)>\frac{3}{2}$ the analysis at the end of Section IV shows that the leading order state dependent terms in the 
energy-momentum tensor will generally grow exponentially with time. This is illustrated in Fig. 6. The exponential growth that occurs here is similar to the well known one for the classical scalar field when $m$ and $\xi$ have values such that $\nu>\frac{3}{2}$ [38, 39]. Such fields are tachyonic and presumbably of little physical interest unless interactions are added to stabilize them.

\section{INFRARED SCALING AND THE GENERALIZED ANOMALY}

We have found that for all $\Re(\nu)<\frac{3}{2}$ the renormalized expectation value of $\left\langle T_{a b}\right\rangle$ approaches the de Sitter invariant Bunch-Davies value for any physically admissable initial state, whereas it approaches the de Sitter invariant AllenFolacci value for any physically admissable initial state in the massless, minimally coupled case. Since all the initial state dependence vanishes asymptotically, these state independent de Sitter invariant fixed point values for $\left\langle T_{a b}\right\rangle_{r e n}$ must be purely geometrical in origin. Indeed, both the Bunch-Davies point-splitting calculation and the Hadamard calculation of Allen-Folacci rely only on the properties of the two-point function of the scalar field $G\left(x, x^{\prime}\right)$ for $x^{\prime} \rightarrow x$. Hence, the BD and AF asymptotic values of $\left\langle T_{a b}\right\rangle_{\text {ren }}$ are certainly "pseudo-local" in the terminology of Ref. [40, i.e. they are expressible in terms of purely local functions of the RW scale factor $a(\eta)$ and its derivatives.

If we specialize now to zero mass, $m=0$, then on simple dimensional grounds the asymptotic $\left\langle T_{a b}\right\rangle_{r e n}$ can be expressed purely in terms of local conserved tensors of fourth adiabatic order. Although we have used adiabatic subtraction methods to renormalize $\left\langle T_{a b}\right\rangle$ it is known that the value of $\left\langle T_{a b}\right\rangle_{r e n}$ so obtained is equal to that in a fully covariant procedure such as dimensional regularization or covariant point-splitting [27,28]. In a fully covariant procedure, which yields a local conserved tensor of fourth adiabatic order, only the Riemann tensor together with its covariant derivatives and contractions can appear. Hence $\left\langle T_{a b}\right\rangle_{r e n}$ for $m=0$ must be expressible entirely in terms of such local geometrical tensors.

In four dimensions the only such local tensors are linear combinations of ${ }^{(1)} H_{a b},{ }^{(2)} H_{a b}$, and ${ }^{(3)} H_{a b}$, where

$$
\text { (1) } \begin{aligned}
H_{a b} & \equiv \frac{1}{\sqrt{-g}} \frac{\delta}{\delta g^{a b}} \int \sqrt{-g} R^{2} \mathrm{~d}^{4} x \\
& =2 g_{a b} \square R-2 \nabla_{a} \nabla_{b} R+2 R R_{a b}-\frac{1}{2} g_{a b} R^{2},
\end{aligned}
$$

vanishes in de Sitter spacetime and

$$
{ }^{(3)} H_{a b}=R_{a}^{c} R_{c b}-\frac{2}{3} R R_{a b}-\frac{1}{2} R_{c d} R^{c d} g_{a b}+\frac{1}{4} R^{2} g_{a b} .
$$

In RW spacetimes, which are all conformally flat, the tensor ${ }^{(2)} H_{a b}$ is proportional to ${ }^{(1)} H_{a b}$ and hence vanishes as well. Therefore the only non-trivial fourth order conserved geometrical tensor in de Sitter spacetime is ${ }^{(3)} H_{a b}$ and we conclude that the fixed point $\mathrm{BD}$ and $\mathrm{AF}$ values found in our previous analysis are proportional to

$$
{ }^{(3)} H_{a b}=\frac{R^{2}}{48} g_{a b}=3\left(\frac{R}{12}\right)^{2} g_{a b} .
$$

Furthermore, since

$$
{ }^{(3)} H_{a b} g^{a b}=-R_{a b} R^{a b}+\frac{R^{2}}{3}=\frac{1}{2}\left(R_{a b c d} R^{a b c d}-4 R_{a b} R^{a b}+R^{2}\right) \equiv \frac{G}{2} \text {, }
$$

in RW spacetimes (where the Weyl tensor vanishes), the coefficient of ${ }^{(3)} H_{a b}$ is proportional to the coefficient of the Gauss-Bonnet integrand in the trace of $\left\langle T_{a b}\right\rangle$. Such a term in the trace is known to correspond to a non-local but nevertheless fully covariant action and this action is precisely the same as that generated by the trace anomaly of free conformal fields [20]. Since we have obtained fixed point results for the asymptotic values of $\left\langle T_{a b}\right\rangle_{\text {ren }}$ for massless fields in de Sitter space which are purely geometrical and proportional to ${ }^{(3)} H_{a b}$, even for non-conformal massless fields, we can give a definite meaning to the value of the proportionality coefficient and the non-local anomaly-like term in the effective action even when $\xi \neq \frac{1}{6}$.

Let us define the generalized anomaly coefficient by fixing the normalization

$$
\lim _{t \rightarrow \infty}\left\langle T_{a b}\right\rangle_{r e n}=-\frac{Q^{2}}{16 \pi^{2}}{ }^{(3)} H_{a b}=-\frac{3 Q^{2}}{16 \pi^{2}}\left(\frac{R}{12}\right)^{2} g_{a b}
$$


With this normalization we find from the asymptotic value of $\left\langle T_{a b}\right\rangle_{r e n}$ for a scalar field in de Sitter space that

$$
Q^{2}=\left\{\begin{array}{l}
Q_{B D}^{2}=\frac{1}{180}-\frac{1}{6}(6 \xi-1)^{2}, \quad m=0, \quad \xi>0, \\
Q_{A F}^{2}=\frac{1}{180}-\frac{1}{6}-\frac{1}{2}=-\frac{119}{180}, \quad m=0, \quad \xi=0 .
\end{array}\right.
$$

The value of $Q^{2}$ for a conformally invariant field $\left(m=0, \xi=\frac{1}{6}\right)$ is $\frac{1}{180}$ and corresponds to the pure trace anomaly coefficient. The first member of (6.6) provides the generalization of this coefficient away from the conformal case. The discontinuous behavior at $\xi=0$ has been discussed in Ref. [19]. As we have seen it arises from the singular behavior of the spatially constant zero mode of the massless, minimally coupled field, which is non-oscillatory and hence cannot be quantized as a Fock mode in the same fashion as the oscillatory modes. We discussed this discontinuity in detail in Section IV.

The connection of the tensor ${ }^{(3)} H_{a b}$ with the trace anomaly may be seen from the general form of the effective action for the anomaly in a conformally flat space with metric 20]

$$
g_{a b}=e^{2 \sigma} \bar{g}_{a b},
$$

namely

$$
S_{\text {eff }}=-\frac{Q^{2}}{16 \pi^{2}} \int \mathrm{d}^{4} x \sqrt{-\bar{g}}\left[\sigma \bar{\Delta}_{4} \sigma+\frac{1}{2}\left(\bar{G}-\frac{2}{3} \overline{.09 .09} \bar{R}\right) \sigma\right]
$$

where $\Delta_{4}=\square^{2}+2 R^{a b} \nabla_{a} \nabla_{b}-\frac{2}{3} R \square^{2}+\frac{1}{3}\left(\nabla^{a} R\right) \nabla_{a}$ is the unique fourth order differential operator acting on scalars which is conformally covariant. A fully covariant but non-local form of the effective action (6.8) can be obtained by solving $\sqrt{-g}\left(G-\frac{2}{3} \square R\right)=\sqrt{-\bar{g}}\left(\bar{G}-\frac{2}{3} \bar{\square} \bar{R}\right)+4 \sqrt{-\bar{g}} \bar{\Delta}_{4} \sigma$ for $\sigma$. In that non-local form all reference to the separation of the metric into background and conformal factor as in (6.7) disappears.

The energy-momentum tensor following from the variation of the local form of the effective action (6.8) with respect to the background metric $\bar{g}_{a b}$, is given by Eq. (2.9) of Ref. [20]. The form of this energy-momentum tensor simplifies considerably on the Einstein space $R \times S^{3}$, where $\bar{G}=\overline{.09 .09 R}=0$. If we set $\sigma=\log a(\eta)$ then this is equivalent to evaluating the components of this energy-momentum tensor in a general RW spacetime with closed spatial sections $(\kappa=+1)$. Using the expressions for ${ }^{(1)} H_{a b}$ and ${ }^{(3)} H_{a b}$ in a general RW space in terms of $a(\eta)$ and its derivatives, we quickly find that

$$
T_{a b}[\sigma]=-\frac{2}{\sqrt{-g}} \frac{\delta S_{\mathrm{eff}}}{\delta \bar{g}^{a b}}=\frac{Q^{2}}{16 \pi^{2}}\left[\frac{1}{18}{ }^{(1)} H_{a b}-{ }^{(3)} H_{a b}+\left.{ }^{(3)} H_{a b}\right|_{R \times S^{3}}\right] .
$$

Hence the tensor ${ }^{(3)} H_{a b}$ which is called "accidentally conserved" in Ref. [1] is associated in fact with the existence of a non-local covariant effective action related to the trace anomaly, which has the local form (6.8) when the metric is conformally decomposed as in (6.7).

The last term in (6.9) would not have been present had we varied the fully covariant but non-local form of the anomalous effective action, in which all reference to the background $\bar{g}_{a b}$ drops out. Equivalently, it is just canceled if we add to (6.9) the Casimir energy on $R \times S^{3}$, which is determined by the anomaly by a further conformal transformation from flat space [41]. In either case, we should drop this last term in (6.9) which depends on the arbitrary background $\bar{g}_{a b}$. Evaluating the first two terms on de Sitter space we find that it is exactly the value of the asymptotic form of the renormalized energy-momentum tensor $\left\langle T_{a b}\right\rangle$ in de Sitter space (6.5), found previously, with the value of $Q^{2}$ for the massless scalar given by (6.6). Thus, the effective action (6.8), associated with the conformal trace anomaly, appears in the effective action for a massless scalar field, even for non-conformal couplings, $\xi \neq \frac{1}{6}$.

Since it is associated with the anomaly, the physical significance of $S_{\text {eff }}$ in the quantum effective action for the scalar field is that it determines the scaling behavior of the field theory under global Weyl transformations of the background space. Using the fact that the Euclidean effective action is given by $I_{\text {eff }}=-S_{\text {eff }}$ and that the Euclidean continuation of de Sitter space is $S^{4}$ with Euler number $\chi=2$, we can vary $I_{\text {eff }}$ with respect to a constant $\sigma=\sigma_{0}$ and obtain

$$
\frac{\partial I_{\mathrm{eff}}}{\partial \sigma_{0}}=\alpha \frac{\mathrm{d} I_{\mathrm{eff}}}{\mathrm{d} \alpha}=\frac{Q^{2}}{32 \pi^{2}} \int_{S^{4}} \mathrm{~d}^{4} x \sqrt{g} G=Q^{2} \chi=2 Q^{2},
$$

since $\frac{\mathrm{d}}{\mathrm{d} \sigma_{0}}=\frac{\mathrm{d}}{\mathrm{d} \log \alpha}$ is a global rescaling of the $S^{4}$. As a check, this relation can be verified explicitly by the $\zeta$ function evaluation of the Euclidean effective action,

$$
I_{\mathrm{eff}}=\frac{1}{2} \operatorname{Tr} \log \left(-.09 .09+m^{2}+\xi R\right)=-\left.\frac{1}{2} \frac{\mathrm{d} \zeta}{\mathrm{d} s}\right|_{s=0}
$$


where the generalized zeta function for the Euclidean continuation of the wave operator appearing in the $\operatorname{Tr} \log$ is

$$
\zeta(s)=\sum_{n=0}^{\infty} d_{n} \lambda_{n}^{-s},
$$

in terms of its eigenvalues $\lambda_{n}$ with degeneracy $d_{n}$ on $S^{4}$. This sum is convergent for $\Re(s)>2$ and defines a meromorphic function of $s$ which is analytic near $s=0$, where its derivative is required. Introducing a mass scale $\mu$ to keep $\zeta(s)$ dimensionless for all $s$ leads in a standard calculation to 42]

$$
-\left.\frac{1}{2} \frac{\mathrm{d} \zeta}{\mathrm{d} s}\right|_{s=0}=-\zeta(0) \log (\mu \alpha)+I_{1}(\nu)
$$

where $I_{1}(\nu)$ is a certain finite function of $\nu$, which from the definition of $\nu$ (3.4) becomes independent of $\alpha$ when $m=0$, and the value $\zeta(0)$ is given by 42 ]

$$
-\zeta(0)=\frac{1}{12}\left(-\nu^{4}+\frac{1}{2} \nu^{2}+\frac{17}{240}\right)=\frac{1}{90}-\frac{1}{3}(6 \xi-1)^{2},
$$

when $m=0$. By making use of (6.6), (6.11), and (6.13), we find that

$$
\alpha \frac{\mathrm{d}}{\mathrm{d} \alpha} I_{\mathrm{eff}}(m=0)=-\zeta(0)=2 Q^{2},
$$

is the behavior of the effective action for a massless scalar field with $\xi>0$ under global Weyl rescaling of the metric, exactly as predicted by(6.10) and the previous discussion based on the anomalous action (6.8).

When $m=0$ and $\xi \rightarrow 0$, the integral representation of the function $I_{1}(\nu)$ develops a logarithmic singularity, which can be traced to the vanishing of the $n=0$ eigenvalue in the expression (6.12) for $\zeta(s)$. In this case the $n=0$ mode must be excluded from the ultraviolet regulated sum over modes, which has the effect of adding one unit to $\zeta(0)$ in the infrared scaling behavior of the effective action [43], and accounts for the addition of $-\frac{1}{2}$ in $Q^{2}$ in the minimally coupled case. Hence the discontinuous behavior of $Q^{2}$ found in (6.6) by our analysis of the asymptotic attractor behavior of the energy-momentum tensor in de Sitter space is precisely the same as that occuring in the effective action under global Weyl rescalings when $m=0$ and $\xi \rightarrow 0$.

Note also that the global Weyl variation is given in terms of the trace of the energy-momentum tensor by

$$
\alpha \frac{\mathrm{d}}{\mathrm{d} \alpha} I_{\mathrm{eff}}(m=0)=-\int_{S^{4}} \mathrm{~d}^{4} x \sqrt{g}\langle T\rangle_{r e n}=-\frac{8 \pi^{2}}{3} \alpha^{4}\langle T\rangle_{r e n} .
$$

Since the $\zeta$ function method is fully covariant, the renormalized trace $\langle T\rangle_{\text {ren }}$ computed in this way cannot contain non-covariant contributions, and must be expressible entirely in terms of local curvature invariants.

Finally we may consider relaxing the condition $m=0$. If $m^{2}>0$, the asymptotic form of the energy-momentum tensor for an arbitrary initial state is given by the BD value. However, if we expand the BD result (3.6) in powers of $R / \mathrm{m}^{2}$, we find that it contains no adiabatic order four $R^{2}$ terms, beginning instead with $R^{3} / \mathrm{m}^{2}$. Mathematically, this is because all terms up to fourth adiabatic order have been removed by the ultraviolet regulating procedure of point splitting or adiabatic subtraction. Hence the coefficient of ${ }^{(3)} H_{a b}$ at asymptotically late times in an arbitrary physical state is given by

$$
Q^{2}=0 \text { for } m^{2}>0,
$$

and no anomalous $S_{\text {eff }}$ term appears in the quantum effective action for a massive field. This is consistent with the interpretation of the anomalous term (6.8) in the effective action of the scalar field as an infrared effect, since the fluctuations of a massive field decouple at large distances or late times, and should induce only strictly irrelevant operators in the effective action in the far infrared, which are suppressed by positive powers of $R / m^{2}$.

Only in the strictly conformal case, $m=0$ and $\xi=\frac{1}{6}$ is the infrared effective action equal to that obtained by ultraviolet methods, such as the $a_{2}$ coefficient in the Schwinger-DeWitt proper time expansion. However, our analysis of the fixed point behavior of $\left\langle T_{a b}\right\rangle_{r e n}$ in de Sitter space shows that its coefficient is connected with the global or extreme infrared scaling of the effective action, and this asymptotic behavior does not depend on the field being conformally invariant. The asymptotic attractor behavior of the energy-momentum tensor in de Sitter space defines an infrared scaling coefficient that reduces to the trace anomaly coefficient in the conformal case, but is a much more general concept than the trace anomaly coefficient, since it is well-defined for all massless fields, conformal or not. It is well-defined even for massive fields, although as (6.17) shows, it vanishes in this case. 


\section{DISCUSSION AND CONCLUSIONS}

In this paper we have considered a quantum scalar field in a fixed de Sitter background. We have studied the late time behavior of the renormalized energy-momentum tensor and have found two important cases in which, for arbitrary physically admissable states, the energy-momentum tensor approaches a particular value at late times. The values approached are those of the energy-momentum tensor in the Bunch-Davies and Allen-Folacci states. Thus, in this sense, these special quantum states behave as fixed point attractors.

In the case $\Re(\nu)<\frac{3}{2}$ we have shown that for all fourth order adiabatic states that are infrared finite the energymomentum tensor approaches the $\mathrm{BD}$ value at late times. The longest time scale for the state dependent terms to redshift away is $\tau=(3-2 \nu)^{-1}$. This has been numerically verified for various values of $m$ and $\xi$ when the fields are in a fourth order adiabatic state. For the case in which $0<\frac{3}{2}-\nu<<1$ and $m$ and $\xi$ are small, we numerically observe a more complicated behavior. The energy-momentum tensor quickly approaches the AF value and grows then linearly with comoving time. Our analytic proof implies that it must then slowly decay to the BD value. It is worth noting that for $\Re(\nu)>0$ the redshift of the state dependent terms in the quantum expectation value of $T_{a b}$ is slower than one might guess from the redshifting of classical matter or radiation, i.e. $a^{-3}$ or $a^{-4}$, respectively.

For the case $\nu=\frac{3}{2}$ we have to distinguish two different possibilities. If the field is massless and minimally coupled then we have proven that, for all fourth order adiabatic states that are infrared finite, the energy-momentum tensor approaches the AF value at late times. This is true for both vacuum and initially populated states. For any other values of $m$ and $\xi$ the renormalized energy-momentum tensor grows linearly with comoving time, indicating that back-reaction effects need to be taken into account. The sign of the linear growth depends on the sign of $\xi$.

For the tachyonic cases $\nu>\frac{3}{2}$ there is no attractor state. Instead, for most values of $m^{2}$ and $\xi$ the renormalized energy-momentum tensor grows like $a^{2 \nu-3}$ at late times for all physically admissable states. Thus back-reaction effects again need to be taken into account.

For the cases in which either the Bunch-Davies or Allen-Folacci states serve as attractors in the above sense and the mass, $m$ of the field is zero, we have shown how these results are connected to the appearance of a certain non-local term in the quantum effective action for the scalar field. This term gives rise to the local geometrical tensor ${ }^{(3)} H_{a b}$ in the asymptotic form of $\left\langle T_{a b}\right\rangle$ at late times, and also determines the global scaling behavior of the effective action for massless fields. Determining this scaling behavior and relating it to the asymptotic $\left\langle T_{a b}\right\rangle$ in de Sitter space has allowed us to generalize the notion of the trace anomaly to massless, non-conformally coupled scalar fields, in the sense that the coefficient of this non-local term in the effective action is well defined even for $\xi \neq \frac{1}{6}$.

The interplay between the UV and IR properties of the state and the mode sums contributing to the energymomentum tensor is a theme running through all of these results. We had to be careful to remove the UV divergences from the unrenormalized $\left\langle T_{a b}\right\rangle$ in order to analyze the late time limit. Since we have found state independent de Sitter invariant results in both the $\nu<\frac{3}{2}$ and massless, minimally coupled cases, and since all the state dependence resides in the finite $k$ modes, their form at high $k$ being restricted by the requirement of matching the adiabatic order four vacuum, it is clear that state independent results for $\left\langle T_{a b}\right\rangle_{r e n}$ are possible only because the contribution to the $\mathrm{BD}$ or $\mathrm{AF}$ expectation value comes from arbitrarily large $k$ at very late times. In fact, inspection of the renormalized expectation value of $\left\langle T_{a b}\right\rangle$ expressed as a mode sum, after the fourth order adiabatic subtraction has been made, shows that the finite contribution comes from $k \sim a$, i.e. when the physical wavelength of the mode is of order of the de Sitter horizon. At very late times, this corresponds to arbitrarily large values of the coordinate wave number $k$.

The finite difference between the $\mathrm{BD}$ value and the $\mathrm{AF}$ value in the $m=\xi=0$ case comes entirely from the $k=1$ mode in closed spatial sections, which is a purely IR effect. This leads to a finite discontinuity in the infrared scaling properties of a massless field since the value of the energy-momentum tensor at $m=\xi=0$ is different from its value in the limit $m=0$ and $\xi \rightarrow 0$. The appearance of the ${ }^{(3)} H_{a b}$ tensor and the corresponding non-local action is quite generic for massless fields; its coefficient $Q^{2}$ vanishes only if the mass is non-zero. Hence we should expect that, although they are certainly not conformal, gravitons will also contribute to this same infrared effective action with a finite value of $Q^{2}$, which can be determined in the same way by a background de Sitter calculation of their quantum $\left\langle T_{a b}\right\rangle$ at late times. We plan to present the results of this calculation in a future publication.

It is also interesting to note that the coefficient of the generalized trace anomaly, $Q^{2}$, is not generically positive, in contrast to all the previously known examples of massless conformal fields 444. It appears that the reason for this is that a positive $Q^{2}$ comes from the ultraviolet behavior of $\left\langle T_{a b}\right\rangle$ for conformal fields, while the infrared behavior of non-conformal fields can contribute a negative value. For any $Q^{2} \neq 0$ the new term in the effective action leads to dynamics for the RW scale factor which is quite different from the Einstein theory, and remains to be investigated in a full dynamical back-reaction calculation. 


\section{ACKNOWLEDGMENTS}

Several of us would like to thank V. Sahni for helpful discussions, and the Institute for Nuclear Theory, University of Washington, where some of this work was completed. P. R. A. would like to thank T-8, Los Alamos National Laboratory for its hospitality. This work was supported in part by grant numbers DMR-9403009 and PHY-9800971 from the National Science Foundation. It was also supported in part by contract number W-7405-ENG-36 from the Department of Energy.

[1] See for example, N. D. Birrell and P. C. W. Davies, Quantum Fields in Curved Space, Cambridge University Press, Cambridge, England (1982), and references contained therein.

[2] F. Cooper and E. Mottola, Phys. Rev. D36, 3114 (1987); F. Cooper, S. Habib, Y. Kluger, E. Mottola, J. P. Paz, and P. R. Anderson, ibid 50, 2848 (1994); F. Cooper, S. Habib, Y. Kluger, and E. Mottola, ibid 55, 6471 (1997).

[3] S. Habib, Y. Kluger, E. Mottola, and J. P. Paz, Phys. Rev. Lett. 76, 4660 (1996).

[4] R. M. Wald, Ann. Phys. (N.Y.) 110, 472 (1978).

[5] A. A. Starobinsky, Phys. Lett. 91B, 99 (1980).

[6] M. V. Fischetti, J. B. Hartle, and B. L. Hu, Phys. Rev. D20, 1772 (1979).

[7] P. R. Anderson, Phys. Rev. D28, 271 (1983); D29, 615 (1984); D32, 1302 (1985); D33, 1567 (1986).

[8] S. Wada, Phys. Rev. D31, 2470 (1985).

[9] W.-M. Suen and P. R. Anderson, Phys. Rev. D35, 2940 (1987).

[10] E. T. Tomboulis, Phys. Lett. 70B, 361 (1977).

[11] B. Allen, Phys. Rev. D32, 3136 (1985).

[12] S. Habib, C. Molina-París, and E. Mottola, Phys. Rev. D61, 24010 (2000).

[13] N. A. Chernikov and E. A. Tagirov, Ann. Inst. Henri Poincare A9, 109 (1968).

[14] E. A. Tagirov, Ann. Phys. (N.Y.)76, 561 (1973).

[15] J. S. Dowker and R. Critchley, Phys. Rev. D13, 3224 (1976).

[16] T. S. Bunch and P. C. W. Davies, Proc. R. Soc. A360, 117 (1978).

[17] B. Allen and A. Folacci, Phys. Rev. D35, 3771 (1987).

[18] A. Folacci, J. Math. Phys. 32, 2813 (1991); ibid 32, 2828 (1991).

[19] K. Kirsten and J. Garriga, Phys. Rev. D48, 567 (1993).

[20] I. Antoniadis, P. O. Mazur, and E. Mottola, Phys. Rev. D55, 4770 (1997).

[21] C. W. Misner, K. S. Thorne, and J. A. Wheeler, Gravitation (Freeman, San Francisco, 1973).

[22] T. S. Bunch, J. Phys. A: Math. Gen. 13, 1297 (1980).

[23] L. Parker, Ph. D. thesis, Harvard University, 1966 (Xerox University Microfilms, Ann Arbor, Michigan, No. 73-31244).

[24] L. Parker and S. A. Fulling, Phys. Rev. D 9, 341 (1974).

[25] S. A. Fulling and L. Parker, Ann. Phys. (N. Y.) 87, 176 (1974).

[26] S. A. Fulling, L. Parker, and B. L. Hu, Phys. Rev. D 10, 3905 (1974).

[27] P. R. Anderson and L. Parker, Phys. Rev. D36, 2963 (1987).

[28] N. D. Birrell, Proc. R. Soc. London. B361, 513 (1978).

[29] C. Lüders and J. E. Roberts, Comm. Math. Phys. 134, 29 (1990).

[30] A.-H. Najmi and A. C. Ottewill, Phys. Rev. D32, 1942 (1985).

[31] W. Junker, DESY-95-144, Doctoral Thesis, hep-th/9507097.

[32] J. Lindig, Phys. Rev. D59, 064011 (1999).

[33] P. R. Anderson and W. Eaker, Phys. Rev. D 61024003 (2000).

[34] I. S. Gradshteyn and I. M. Ryzhik, Table of Integrals, Series, and Products (Academic Press, New York, 1980).

[35] Higher Transcendental Functions, Vol. I, Bateman Manuscript Project, A. Erdelyi, ed., McGraw-Hill, New York (1953).

[36] V. Sahni and S. Habib, Phys. Rev. Lett. 81, 1766 (1998).

[37] L. P. Grishchuk, Zh. Eksp. Teor. Fiz 67, 825 (1974) (Sov. Phys. JETP, 40, 409 (1975)).

[38] A. D. Dolgov, in The Very Early Universe, edited by G. W. Gibbons, S. W. Hawking, and S. T. C. Siklos (Cambridge University Press, Cambridge, 1983).

[39] L. H. Ford, Phys. Rev. D35, 2339 (1987).

[40] P. C. W. Davies, S. A. Fulling, S. M. Christensen, and T. S. Bunch, Ann. Phys. 109, 108 (1977).

[41] L. S. Brown and J. P. Cassidy, Phys. Rev. D16, 1712 (1977).

[42] B. Allen, Nucl. Phys. B226, 228 (1983); P. O. Mazur and E. Mottola, Nucl. Phys. B278, 694 (1986).

[43] I. Antoniadis, P.O. Mazur, and E. Mottola, Nucl. Phys. B388, 627 (1992).

[44] M. J. Duff, Nucl. Phys. B125, 334 (1977). 


\section{APPENDIX A: THE CASES OF IMAGINARY AND INTEGER VALUES OF $\nu$}

In this appendix we show that the proof in Section III works for imaginary values of $\nu$ as well as for $\nu=0,1$. For imaginary values of $\nu$ it is useful to write $\nu=i \gamma$ with $\gamma$ a positive real number. Then the formulas in Eq. (3.23a) and (3.23b) are the same as before. However the values of the $A_{i}$ are different. They are given by

$$
\begin{aligned}
A_{1}= & \frac{\pi}{2 k} e^{-\gamma \pi}\left[\operatorname{coth}(\gamma \pi) \operatorname{csch}(\gamma \pi)\left(\left(1+2 n_{k}\right)\left|c_{2}\right|^{2}+n_{k}\right)-\frac{1}{2} \operatorname{coth}(\gamma \pi) \operatorname{csch}(\gamma \pi)\left(1+2 n_{k}\right)\left(c_{1} c_{2}^{*}+c_{1}^{*} c_{2}\right)\right. \\
& \left.-\frac{1}{2} \operatorname{csch}(\gamma \pi)\left(1+2 n_{k}\right)\left(c_{1} c_{2}^{*}-c_{1}^{*} c_{2}\right)\right], \\
A_{2}= & -\frac{\pi}{k} e^{-\gamma \pi}\left[\left(1+\operatorname{csch}^{2}(\gamma \pi)\left(\left(1+2 n_{k}\right)\left|c_{2}\right|^{2}+n_{k}\right)-\frac{1}{2} \operatorname{csch}^{2}(\gamma \pi)\left(1+2 n_{k}\right)\left(c_{1} c_{2}^{*}+c_{1}^{*} c_{2}\right)\right],\right. \\
A_{3}= & \frac{\pi}{2 k} e^{-\gamma \pi}\left[\operatorname{coth}(\gamma \pi) \operatorname{csch}(\gamma \pi)\left(\left(1+2 n_{k}\right)\left|c_{2}\right|^{2}+n_{k}\right)-\frac{1}{2} \operatorname{coth}(\gamma \pi) \operatorname{csch}(\gamma \pi)\left(1+2 n_{k}\right)\left(c_{1} c_{2}^{*}+c_{1}^{*} c_{2}\right)\right. \\
& \left.+\frac{1}{2} \operatorname{csch}(\gamma \pi)\left(1+2 n_{k}\right)\left(c_{1} c_{2}^{*}-c_{1}^{*} c_{2}\right)\right] .
\end{aligned}
$$

The last terms in the expressions for $A_{1}$ and $A_{3}$ are imaginary. Substitution into Eqs. (3.23a) and (3.23b) shows that the contributions of these terms to the energy-momentum tensor cancel.

Noting that $\nu$ is imaginary, one sees that the argument for the vanishing of the first integral in Eq. (3.27) is unchanged. Since the terms in the integrand are being bounded for the second integral one must take the real part of $\beta_{i}$ and substitute that for $\beta_{i}$ in Eqs. (3.28) and (3.31). After making this substitution it is still the case that each term in (3.31) vanishes in the limit $\eta \rightarrow 0^{-}$. The argument for the vanishing of the third integral in Eq.(3.27) is unchanged when $\nu$ is imaginary. Thus, the proof is valid for imaginary values of $\nu$.

For $\nu=n=0,1$ we use the identity

$$
\begin{aligned}
& H_{n}^{(1)}(z)=J_{n}(z)+i N_{n}(z) \\
& H_{n}^{(2)}(z)=J_{n}(z)-i N_{n}(z)
\end{aligned}
$$

and then use the well known power series solutions for $N_{0}$ and $N_{1}$ to write

$$
N_{n}(z)=\frac{2}{\pi} J_{n}(z) \log (z)+P_{n}(z)
$$

where $P_{n}(z)$ is a series of the form

$$
P_{n}(z)=\sum_{j=0}^{\infty} b_{n j} z^{2 j-n}
$$

Because of the $\log (z)$ term it is useful to write

$$
\frac{\mathrm{d} N_{n}(z)}{\mathrm{d} z}=\frac{2}{\pi} \frac{\mathrm{d} J_{n}(z)}{\mathrm{d} z} \log (z)+Q_{n}(z),
$$

with $Q_{n}(z)$ a series of the form

$$
Q_{n}(z)=\sum_{j=0}^{\infty} \epsilon_{n j} z^{2 j-n-1} .
$$

We can conclude that Eqs. (3.23a) and 3.23b remain the same but with different expressions for $A_{i}, S_{i}$, and $\beta_{i}$. The new expressions for the $A_{i}$ are given by

$$
\begin{aligned}
A_{1}= & -\frac{\pi}{2 k}\left[\left(1+\frac{4}{\pi^{2}}(\log z)^{2}\right)\left(\left(1+2 n_{k}\right)\left|c_{2}\right|^{2}+n_{k}\right)+\frac{1}{2}\left(1-\frac{4}{\pi^{2}}(\log z)^{2}\right)\left(1+2 n_{k}\right)\left(c_{1} c_{2}^{*}+c_{1}^{*} c_{2}\right)\right. \\
& \left.+\frac{2 i}{\pi} \log z\left(1+2 n_{k}\right)\left(c_{1} c_{2}^{*}-c_{1}^{*} c_{2}\right)\right], \\
A_{2}= & -\frac{\pi}{k}\left[\frac{2}{\pi} \log z\left(\left(1+2 n_{k}\right)\left|c_{2}\right|^{2}+n_{k}\right)-\frac{1}{\pi} \log z\left(1+2 n_{k}\right)\left(c_{1} c_{2}^{*}+c_{1}^{*} c_{2}\right)+\frac{i}{2}\left(1+2 n_{k}\right)\left(c_{1} c_{2}^{*}-c_{1}^{*} c_{2}\right)\right], \\
A_{3}= & -\frac{\pi}{2 k}\left[\left(\left(1+2 n_{k}\right)\left|c_{2}\right|^{2}+n_{k}\right)-\frac{1}{2}\left(1+2 n_{k}\right)\left(c_{1} c_{2}^{*}+c_{1}^{*} c_{2}\right)\right] .
\end{aligned}
$$


Note that the $A_{i}$ are now functions of both $k$ and $\log (z)$. The new expressions for the $\beta_{i}$ and $S_{i}$ are given in Table 2 .

\begin{tabular}{|r||c|c|}
\hline \multicolumn{1}{|c||}{} & $\beta_{i}$ & $S_{i}$ \\
\hline \hline 1 & $5+2 n$ & $z^{5} J_{n}^{2}(z)$ \\
\hline 2 & 5 & $z^{5} J_{n}(z) P_{n}(z)$ \\
\hline 3 & $5-2 n$ & $z^{5} P_{n}^{2}(z)$ \\
\hline 4 & $3+2 n$ & $z^{3} J_{n}^{2}(z)$ \\
\hline 5 & 3 & $z^{3} J_{n}(z) P_{n}(z)$ \\
\hline 6 & $3-2 n$ & $z^{3} P_{n}^{2}(z)$ \\
\hline 7 & $3+2 n$ & $z^{4} \frac{\mathrm{d}}{\mathrm{d} z} J_{n}^{2}(z)$ \\
\hline 8 & 3 & $\left.z^{4}\left(\frac{\mathrm{d}}{\mathrm{d} z} J_{n}(z)\right) P_{n}(z)+J_{n}(z) Q_{n}(z)\right)$ \\
\hline 9 & $3-2 n$ & $2 z^{4} P_{n}(z) Q_{n}(z)$ \\
\hline 10 & $3+2 n$ & $z^{5}\left(\frac{\mathrm{d}}{\mathrm{d} z} J_{n}(z)\right)^{2}$ \\
\hline 11 & 3 & $z^{5}\left(\frac{\mathrm{d}}{\mathrm{d} z} J_{n}(z)\right) Q_{n}(z)$ \\
\hline 12 & $3-2 n$ & $z^{5} Q_{n}^{2}(z)$ \\
\hline
\end{tabular}

Table 2

Substitution these expressions into Eqs. (3.23a) and (3.23b) shows that for the first integral in (3.27) the expressions are of the same form, except that some terms have factors of $\log (z)$. However these do not prevent the terms in the first integral on the right hand side of (3.27) from vanishing asymptotically. For the second integral there are still terms of the form given in Eq. (3.28). However, some of them also have factors $\operatorname{of} \log (z)$. Inserting factors of $\log (z)$ into Eq. (3.31) and computing the integrals, one finds that the terms all vanish in the limit $\eta \rightarrow 0^{-}$. The factors of $\log (z)$ also do not affect the asymptotic vanishing of the third integral on the right in Eq. (3.27).

Therefore, in all cases where $\Re(\nu)<\frac{3}{2}$ the quantity $\left\langle T_{a b}\right\rangle_{S D}$ vanishes in the limit $\eta \rightarrow 0^{-}$.

\section{APPENDIX B: THE HARMONIC OSCILLATOR WITH $\omega \rightarrow 0$}

In this Appendix we give a detailed discussion of the harmonic oscillator with vanishing frequency, pointing out the analogy with the $\nu \rightarrow \frac{3}{2}$ limit in de Sitter space. Consider a simple harmonic oscillator with Hamiltonian

$$
H=\frac{1}{2} \dot{\phi}^{2}+\frac{1}{2} \omega^{2} \phi^{2}
$$

The single degree of freedom $\phi$ can be quantized by introducing the operator representation

$$
\phi(t)=\langle\phi(t)\rangle+a \psi(t)+a^{\dagger} \psi^{*}(t)
$$

where $a^{\dagger}$ and $a$ are creation and destruction operators obeying

$$
\left[a, a^{\dagger}\right]=1
$$

The canonical commutation relations $[\phi, \dot{\phi}]=i$ are satisfied provided the mode function $\psi$ obeys the Wronskian condition (2.8). The equation of motion for the Heisenberg operator $\phi(t)$ implies that the mode functions also satisfy 


$$
\ddot{\psi}+\omega^{2} \psi=0
$$

In order to satisfy the Wronskian condition we choose the fundamental normalized complex solution to this equation to be

$$
f(t) \equiv \frac{1}{\sqrt{2 \omega}} e^{-i \omega t} .
$$

The general solution satisfying both the Wronskian condition and (B4) can be written as the linear combination

$$
\psi(t)=\alpha f(t)+\beta f^{*}(t)
$$

where

$$
|\alpha|^{2}-|\beta|^{2}=1
$$

Thus, up to an irrelevant overall phase, the Bogoliubov coefficients can be parameterized in terms of two real parameters in the form

$$
\begin{aligned}
& \alpha=\cosh \theta \\
& \beta=\sinh \theta e^{-i \delta} .
\end{aligned}
$$

We could keep the c-number expectation value $\langle\phi(t)\rangle$ non-zero in general, which would lead to the general Gaussian state with displaced origin that we considered in some earlier papers [3. Since we do not require it in our free field de Sitter calculation, we specialize to the case where $\langle\phi(t)\rangle=\langle\dot{\phi}(t)\rangle=0$ in the following. With this restriction the field operator can be written simply as

$$
\phi(t)=a \psi(t)+a^{\dagger} \psi^{*}(t)
$$

We make use of the freedom in the $\alpha$ and $\beta$ coefficients to require

$$
\begin{aligned}
\langle a a\rangle & =\left\langle a^{\dagger} a^{\dagger}\right\rangle=0, \\
\left\langle a a^{\dagger}\right\rangle & =\left\langle a^{\dagger} a\right\rangle+1=\frac{\sigma+1}{2},
\end{aligned}
$$

with no loss of generality. The positive real parameter $\sigma \geq 1$ has the interpretation $\sigma=1+2 n$, with $n$ the average number of "particles" in the $a$ basis. Thus we need the three parameters $\theta, \delta$, and $\sigma$ to specify the general semi-classical (coherent) state of the field.

Using the definitions above it now follows that the average energy in this state is

$$
E=\langle H\rangle=\frac{\omega}{2} \sigma\left(1+2|\beta|^{2}\right)
$$

Note that we have not insisted that the state be an eigenstate of the number operator $a^{\dagger} a$ so $n$ is only the average number of particles in the state, which can take on any non-negative value and need not be an integer. If $n>0$ and the state is a Gaussian, consistent with all connected correlations vanishing except the two-point function, then it turns out that the state is necessarily a mixed state [3].

We are interested in the nature of the "vacuum" state in the limit $\omega \rightarrow 0$, i.e. when our single degree of freedom becomes that of a free particle. In this limit we can no longer retain the complex oscillating mode function basis since their normalization diverges due to the $(2 \omega)^{-\frac{1}{2}}$ factor in (B5). However, the mode equation $\ddot{\psi}=0$ clearly possesses the regular real solutions $u=1$ and $v=t$, which we can obtain from the complex oscillatory solutions by taking appropriate linear combinations in the limit of vanishing frequency, i.e.

$$
\begin{aligned}
& u(t) \equiv \lim _{\omega \rightarrow 0}\left\{\sqrt{\frac{\omega}{2}}\left(f+f^{*}\right)\right\}=1, \\
& v(t) \equiv i \lim _{\omega \rightarrow 0}\left\{\frac{1}{\sqrt{2 \omega}}\left(f-f^{*}\right)\right\}=t .
\end{aligned}
$$

These definitions are analogous to Eq. (4.7) of the text, with $\omega$ replacing $3-2 \nu$.

The general linear combination of modes can then be rewritten in the form 


$$
\psi(t)=\frac{(\alpha+\beta)}{\sqrt{2 \omega}} u-i \frac{\sqrt{\omega}}{2}(\alpha-\beta) v=B u+A v \rightarrow A t+B
$$

where the quantities

$$
\begin{aligned}
& A \equiv-i \lim _{\omega \rightarrow 0}\left\{\sqrt{\frac{\omega}{2}}(\alpha-\beta)\right\}, \\
& B \equiv \lim _{\omega \rightarrow 0}\left\{\frac{1}{\sqrt{2 \omega}}(\alpha+\beta)\right\},
\end{aligned}
$$

are analogous to those defined by (4.8) of the text. They also satisfy

$$
A^{*} B-B^{*} A=i\left(|\alpha|^{2}-|\beta|^{2}\right)=i .
$$

We can also define the time-independent Hermitian operators

$$
\begin{aligned}
Q & \equiv \frac{1}{\sqrt{2 \omega}}\left[(\alpha+\beta) a+(\alpha+\beta)^{*} a^{\dagger}\right] \rightarrow B a+B^{*} a^{\dagger}, \\
P & \equiv-i \sqrt{\frac{\omega}{2}}\left[(\alpha-\beta) a-(\alpha-\beta)^{*} a^{\dagger}\right] \rightarrow A a+A^{*} a^{\dagger},
\end{aligned}
$$

analogous to (4.11) in the de Sitter case. They obey the canonical commutation relations

$$
[Q, P]=i,
$$

so that in the limit $\omega \rightarrow 0$

$$
\phi(t) \rightarrow u(t) Q+v(t) P=Q+t P,
$$

and in the same limit

$$
E=\frac{1}{2}\left\langle P^{2}\right\rangle=\frac{\sigma}{4} \lim _{\omega \rightarrow 0}\left\{\omega|\alpha-\beta|^{2}\right\}=\frac{\sigma}{2}|A|^{2} .
$$

Since $\sigma$ is just an overall factor and we are interested in pure vacuum-like states, we can set $\sigma=1$.

We see that depending on exactly how we take the limit $\omega \rightarrow 0$, we can get many different values for the energy. If we tried to keep the pure positive frequency solution $\psi=f$, i.e. $\alpha=1$ and $\beta=0$, then the energy would vanish in the limit $\omega \rightarrow 0$. However, by computing the correlation function, $\left\langle\phi(t) \phi\left(t^{\prime}\right)\right\rangle$, we would find that the correlator diverges in this limit. In our simple example it is clear why. The ordinary ground state vacuum wave function of the simple harmonic oscillator is a Gaussian with a width proportional to $\omega^{-\frac{1}{2}}$. Hence $\left\langle Q^{2}\right\rangle \sim \omega^{-1}$ in this state, which becomes non-normalizable in the limit, unless we were to put the particle in a box of finite volume. But this option is unavailable to us if the "particle" is a mode of a field and the range of the field variable $\phi$ is $(-\infty, \infty)$. Thus, we must reject the positive frequency harmonic oscillator vacuum in the limit $\omega \rightarrow 0$ since it is a non-normalizable state.

In order for the state to remain normalizable and the mode functions bounded, the quantities $A$ and $B$ must remain finite in the limit of vanishing $\omega$. Inspection of the definitions (B14) shows that this requires that the original Bogoliubov coefficients $|\alpha|$ and $|\beta|$ go to infinity. In fact, using (B8) in the definitions of $A$ and $B$ a simple calculation shows that the Bogoliubov parameters $\theta$ and $\delta$ must behave like

$$
\begin{aligned}
& \theta \rightarrow-\frac{1}{2} \log \left(\frac{\omega}{2}\right)+\log |A|, \\
& \delta \rightarrow \pi+\omega T
\end{aligned}
$$

as $\omega \rightarrow 0$, so that

$$
\begin{aligned}
& A=-i|A|, \quad \text { and } \\
& B=\frac{1}{2|A|}+\frac{i}{2}|A| T,
\end{aligned}
$$

remain finite in that limit. Thus, the two complex numbers $A$ and $B$ obeying $(\overline{B 15})$ and the state they characterize can be specified by the two real numbers $|A| \geq 0$ and $T$, up to an unobservable overall phase. Comparison of these 
expressions with the corresponding equations (4.7) and (4.13) in the paper by Allen and Folacci [17] shows that our $A$ here is equivalent to Allen and Folacci's $2 A$. This is because of a factor of 2 difference in the normalization of their time dependent mode $v(t)$.

Since $\theta \rightarrow \infty$, in a sense the normalizable "vacuum" state with finite $\left\langle P^{2}\right\rangle=|A|^{2}$ and finite $\left\langle Q^{2}\right\rangle=|B|^{2}$ is infinitely far from the usual ground state vacuum of the harmonic oscillator, and is not an eigenstate of the free particle Hamiltonian at $\omega=0$. Instead, it is just a Gaussian state centered at $\langle Q\rangle=0$ and $\langle P\rangle=0$ with normalized wave function [3]

$$
\Psi(q)=\frac{1}{\left(2 \pi|B|^{2}\right)^{\frac{1}{4}}} \exp \left(-\frac{|A|}{2 B^{*}} q^{2}\right)
$$

in the position representation where $Q \Psi=q \Psi$ and $P \Psi=-i \frac{\mathrm{d}}{\mathrm{d} q} \Psi$. This state is time reversal invariant if and only if $T=0$, in which case $B=\frac{1}{2|A|}$ becomes real. On the other hand, an eigenstate of the free Hamiltonian $P^{2} / 2$ is necessarily non-normalizable since diagonalizing $P$ requires infinite spread in $Q$. These remarks carry over equally well to the $k=1$ mode in the de Sitter case.

There is no reason to diagonalize $H$ in the field theory case as Allen and Folacci do in their Eqs. (4.26) to (4.28) [17], since the energy-momentum tensor is time dependent in general, and back-reaction has been neglected in our simple calculations. Diagonalizing just the matter Hamiltonian without taking into account the metric is no better than a leading order mean field approximation to the much more difficult full problem with metric fluctuations taken into account. Given that what we are doing is only a semi-classical approximation to this full problem in any case, what makes the most sense from our perspective is to take well-defined normalizable initial adiabatic states for the scalar field and allow them to evolve as they will. Our initial state trial wave functional B22 then remains a bounded Gaussian functional, consistent with semi-classical mean field methods (although it may well spread over time depending on the dynamics).

As should be clear, the non-normalizable positive frequency vacuum with $\alpha=1$ and $\beta=0$ is analogous to the Bunch-Davies vacuum, while the normalizable state related to it by an infinite Bogoliubov transformation (but only a finite shift in zero point energy) is analogous to the Allen-Folacci states parameterized by $A$ and $B$ or $|A|$ and $T$. However, whereas the energy of the normalizable state is finite and arbitrary, depending on the arbitrary $|A|^{2}$ coefficient in (B19), and the non-normalizable positive frequency state has zero energy in this simple harmonic oscillator example, the converse is true at late times in de Sitter space. This is due to the different kinematics and form of the energy-momentum tensor of the scalar field in the de Sitter case. Comparison of the energy (B19) of the harmonic oscillator example and the corresponding $k=1$ late time energy density in the de Sitter case with $m=0$ and $\xi$ small, viz.

$$
\varepsilon_{1} \rightarrow \frac{1}{2 a^{2}}\left(\frac{\mathrm{d} \phi_{1}}{\mathrm{~d} \eta}\right)^{2}+3 \xi \phi_{1}^{2}=\frac{1}{4 \pi^{2} a^{2}}\left(\frac{\mathrm{d} v}{\mathrm{~d} \eta}\right)^{2} P^{2}+\frac{3 \xi}{2 \pi^{2}}(Q+v P)^{2}
$$

shows that the difference is the redshift factor of $a^{-2}$ multiplying the infrared finite $\left\langle P^{2}\right\rangle$ in the de Sitter case. Thus, whereas this can take on the finite state dependent value $|A|^{2}$ in the harmonic oscillator example, its asymptotic late time value vanishes in de Sitter space when multiplied by $a^{-2}$, and becomes state independent in any infrared finite state. Further, in the simple harmonic oscillator the usual ground state becomes an eigenstate of $P$ and has zero energy as $\omega \rightarrow 0$, since $\omega^{2}\left\langle(Q+v P)^{2}\right\rangle \sim \omega \rightarrow 0$ drops out of the energy in this limit, whereas in the de Sitter case, since $\xi$ goes to zero only linearly with $3-2 \nu$, the Bunch-Davies expectation value receives a finite contribution from the $k=1$ mode from the infinite spread in $\xi\left\langle Q^{2}\right\rangle$ which has no $a^{-2}$ redshift factor in (B23). 